Universidad

BIBLIOTECA

Document downloaded from the institutional repository of the University of Alcala: http://ebuah.uah.es/dspace/

This is a postprint version of the following published document:

Pérez-Díaz, Sonia. 2019, "On the computation of singularities of parametrized ruled surfaces", Advances in Applied Mathematics, vol. 110, pp. $270-298$

Available at https://doi.org/10.1016/i.aam.2019.07.005

(C) 2019 Elsevier

(Article begins on next page)

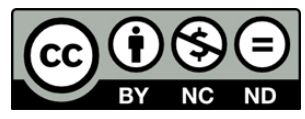

This work is licensed under a

Creative Commons Attribution-NonCommercial-NoDerivatives

4.0 International License. 


\title{
On the Computation of Singularities of Parametrized Ruled Surfaces
}

\author{
Sonia Pérez-Díaz \\ ${ }^{a}$ Dpto. de Física y Matemáticas, Universidad de Alcalá, E-28871 Madrid, Spain
}

\begin{abstract}
Given a ruled surface $\mathcal{V}$ defined in the standard parametric form $\mathcal{P}\left(t_{1}, t_{2}\right)$, we present an algorithm that determines the singularities (and their multiplicities) of $\mathcal{V}$ from the parametrization $\mathcal{P}$. More precisely, from $\mathcal{P}$ we construct an auxiliary parametric curve and we show how the problem can be simplified to determine the singularities of this auxiliary curve. Only one univariate resultant has to be computed and no elimination theory techniques are necessary. These results improve some previous algorithms for detecting singularities for the special case of parametric ruled surfaces.
\end{abstract}

Keywords: ruled surface; standard parametric form; singularities

\section{Introduction}

The study of some classes of surfaces with special properties such as developable, minimal, II-minimal, and II-flats is one of the principal aims of the classical differential geometry. There are many important kinds of surfaces such as cyclic, revolution, helicoid, rotational, canal, ruled surfaces and so on. This kind of surfaces has an important role and many applications in different fields, such as: physics, computer aided geometric design, geometric modeling, design problems in spatial mechanism, etc. In particular, many researches have studied important geometric and algebraic properties concerning the ruled surfaces which are surfaces generated by moving a straight line continuously in the space.

For instance, using the $\mu$-bases method, Chen et al. [9] give an implicitization algorithm for a rational ruled surface. In [8], authors find a simplified reparametrization of a given parametrization of a ruled surface which does not contain any non-generic base point and has a pair of directrices with the lowest possible degree. In [5, 12], authors study the ruled surface using $\mu$-bases. Li et al. [16] compute a proper reparametrization of an improper parametric ruled surface. Andradas et al. [1] present an algorithm to

Email address: sonia.perez@uah.es (Sonia Pérez-Díaz) 
decide whether a proper rational parametrization of a ruled surface can be properly reparametrized over the real field. The ruled surfaces are used for geometric modeling of architectural freeform design in [17]. The collision and intersection of the ruled surfaces are discussed in [10, 28]. Izumiya et al. [15] study the cylindrical helices and Bertrand curves on ruled surfaces. In [26], authors present an algorithm that covers any given rational ruled surface with two rational parametrizations. In [29], authors present algorithms to determine whether a given implicit surface is a rational ruled surface and in the affirmative case, a parametrization (in standard form) of the surface is computed. In addition, after a given rational parametric surface is determined as a ruled one, it is reparameterized to the standard form.

In this paper, we consider a very important research topic: the computation of the singular locus of a parametric ruled surface. The interest on the study of singularities is motivated by multiple reasons, being one of them their applicability; for instance, in geometric modeling, when determining the shape and the topology of curves and of surfaces, etc. When the surface is implicitly defined, the singularities and their multiplicities can be directly computed by applying elimination theory techniques as Gröbner bases. However when the surface is given by means of a rational parametrization, besides the question of computing the singular locus and its multiplicity structure, one has the additional problem of determining the parameter values that generate the singular points with their corresponding multiplicities. This, for instance, can be useful when using a parametrization for plotting a curve or a surface or when utilizing a parametrization for analyzing the intersection variety of two varieties being one of them parametrically given.

The basic idea of the approach presented in this paper is to compute the singularities and their multiplicities of an auxiliary parametric curve directly constructed from the input rational parametrization of the surface. The singularities of this auxiliary curve provide the singularities of the input ruled surface and only a finite set of points have to be additionally analyzed. In this way, the problem is greatly simplified (see Section 5 ) and the algorithm presented is very efficient since it only relies on the computation of a univariate resultant (see [2], [7], [3], [4], [19]).

The structure of the paper is as follows. First, some necessary preliminaries are presented in Section 2. In Section 3, we present the main result, Theorem 10, and the algorithm derived from this theorem that computes all the singularities of $\mathcal{V}$ from $\mathcal{P}$ (see Subsection 3.1). For this purpose, we consider a parametric ruled surface $\mathcal{V}$ defined by a standard reduced form parametrization $\mathcal{P}$ (see equality (2)). From $\mathcal{P}$, we construct an auxiliary parametric curve, and we show how the problem can be simplified to determine the singularities of this auxiliary curve. Using the results obtained in [2] and [19], we show that only a univariate resultant has to be computed and no elimination theory techniques are necessary. Furthermore, algebraic numbers are not introduced during the computations. We illustrate the results with several examples. In order to 
facilitate the reading of the paper, the proofs of the results obtained in Section 3 are presented in Section 4. Finally, we conclude with Section 5, where we argue about the interest of the proposed method and we enumerate topics for further study.

\section{Preliminaries and Previous Results}

In this section we introduce the notation and terminology that will be used throughout the paper. In addition, we recall some basic results on surfaces (see Subsection 2.1) and curves (see Subsection 2.2).

\subsection{Preliminaries on Surfaces: Ruled Surfaces and Singularities}

Let $\mathbb{K}$ be an algebraically closed field of characteristic zero, and $\mathcal{V}$ a rational surface over $\mathbb{K}$. If $\mathcal{V}$ is defined by an irreducible polynomial $F(\bar{x}) \in \mathbb{K}[\bar{x}], \bar{x}=\left(x_{1}, x_{2}, x_{3}\right)$, by applying the results in [29], one may easily check whether $\mathcal{V}$ is a ruled surface, and in the affirmative case, compute a rational parametrization in standard form; i.e. a parametrization of the form

$\mathcal{Q}(\bar{t})=\left(m_{1}\left(t_{1}\right)+t_{2} n_{1}\left(t_{1}\right), m_{2}\left(t_{1}\right)+t_{2} n_{2}\left(t_{1}\right), m_{3}\left(t_{1}\right)+t_{2} n_{3}\left(t_{1}\right)\right) \in \mathbb{K}(\bar{t})^{3}, \quad \bar{t}=\left(t_{1}, t_{2}\right)$

where there exists at least one $i \in\{1,2,3\}$ such that $n_{i} \neq 0$. If the surface $\mathcal{V}$ is defined by a parametrization (not necessarily proper) over $\mathbb{K}$, in [29], it is also analyzed whether $\mathcal{V}$ is a ruled surface, and in the affirmative case a proper reparametrization in standard form is computed.

Note that if $\mathcal{V}$ is defined by $(1)$, and $n_{3} \neq 0$, the surface $\mathcal{V}$ admits a parametrization of the form

$$
\mathcal{P}(\bar{t})=\left(p_{1}(\bar{t}), p_{2}(\bar{t}), p_{3}(\bar{t})\right)=\left(\frac{p_{11}\left(t_{1}\right)+t_{2} p_{12}\left(t_{1}\right)}{q\left(t_{1}\right)}, \frac{p_{21}\left(t_{1}\right)+t_{2} p_{22}\left(t_{1}\right)}{q\left(t_{1}\right)}, t_{2}\right) \in \mathbb{K}(\bar{t})^{3},
$$

where $p_{k 2}\left(t_{1}\right) / q\left(t_{1}\right)=n_{k} / n_{3} \neq 0$, for some $k=1,2$. Such a parametrization is obtained by performing the birational transformation $\left(t_{1}, t_{2}\right) \rightarrow\left(t_{1},\left(t_{2}-m_{3}\left(t_{1}\right)\right) / n_{3}\left(t_{1}\right)\right)$. One may reason similarly as above, if $n_{1} \neq 0$ or $n_{2} \neq 0$. In the following, we assume that $\mathcal{V}$ admits a parametrization of the form (2), and we refer to $\mathcal{P}$ as the standard reduced form parametrization of $\mathcal{V}$.

Under these conditions, we observe that the corresponding projective surface $\overline{\mathcal{V}}$ is defined by the projective parametrization

$$
\overline{\mathcal{P}}(\bar{t})=\left(q\left(t_{1}\right), p_{11}\left(t_{1}\right)+t_{2} p_{12}\left(t_{1}\right), p_{21}\left(t_{1}\right)+t_{2} p_{22}\left(t_{1}\right), t_{2} q\left(t_{1}\right)\right) \in \mathbb{P}^{3}(\mathbb{K}(\bar{t})) .
$$

We represent by $\overline{\mathcal{P}}\left(t_{0}, t_{1}, t_{2}\right)$ the homogeneous parametrization of $\overline{\mathcal{V}}$ obtained from the homogenization of $\overline{\mathcal{P}}\left(t_{1}, t_{2}\right)$. Finally, we observe that $\overline{\mathcal{V}}$ is defined by the homogenization $\bar{F}\left(x_{0}, x_{1}, x_{2}, x_{3}\right)$ of $F\left(x_{1}, x_{2}, x_{3}\right)$. Therefore, if we write $F(\bar{x})=F_{d}(\bar{x})+F_{d-1}(\bar{x})+$ 
$\cdots+F_{0}(\bar{x}), \bar{x}=\left(x_{1}, x_{2}, x_{3}\right)$, where $F_{k}(\bar{x})$ is a homogeneous polynomial of degree $k$, and $F_{d} \neq 0$, then $\bar{F}\left(x_{0}, \bar{x}\right)=F_{d}(\bar{x})+F_{d-1}(\bar{x}) x_{0}+\cdots+F_{0}(\bar{x}) x_{0}^{d}$.

From the results of [26], we know that every rational ruled surface can be covered with at most two surface parametrizations. In fact, every rational ruled surface can be parametrized in an standardized way that misses at most one line. This line can be computed by applying Theorem 2.6 in [26].

In this paper, we present a method for computing the singularities (and multiplicities) of $\overline{\mathcal{V}}$ from the standard reduced form parametrization (2) defining $\overline{\mathcal{V}}$ (note that the affine singularities of $\mathcal{V}$ are exactly the affine singularities of $\overline{\mathcal{V}}$ ). Taking into account the above sentence, we additionally will have to analyze the missed line or to consider a new surface standard reduced form parametrization, and to apply again the algorithm.

In the following, some preliminaries concerning singular points and multiplicities are introduced.

Let $P=\left(a_{1}, a_{2}, a_{3}\right) \in \mathcal{V}$. We say that $P$ is a point of multiplicity $m$ on $\mathcal{V}$ if and only if all the derivatives of $F$ up to and including the $(m-1)$-th vanish at $P$ but at least one $m$-th derivative does not vanish at $P$. We denote it by $\operatorname{mult}(P, \mathcal{V})$.

$P$ is called a simple point on $\mathcal{V}$ if and only if the multiplicity is 1 . Otherwise, we say that $P$ is a multiple or singular point (or singularity) of multiplicity $m$ on $\mathcal{V}$ or an $m$-fold point.

Observe that the multiplicity of $\mathcal{V}$ at $P$ is given as the order of the Taylor expansion of $F$ at $P$. The tangents to $\mathcal{V}$ at $P$ are the irreducible factors of the first non-vanishing form in the Taylor expansion of $F$ at $P$, and the multiplicity of a tangent is the multiplicity of the corresponding factor. If all the $m$ tangents at the $m$-fold point $P$ are different, then this singularity is called ordinary, and non-ordinary otherwise.

As far as projective surfaces are concerned all these definitions also apply, since every point at infinity can be transformed to a point at finite distance by a change of coordinates. In addition, we note that $\operatorname{mult}\left(\left(a_{1}: a_{2}: a_{3}: 1\right), \overline{\mathcal{V}}\right)=\operatorname{mult}(P, \mathcal{V})$.

Given an affine point $P=\left(a_{1}, a_{2}, a_{3}\right) \in \mathbb{K}^{3}$, in the following we summarize some results that allow us to compute $\operatorname{mult}(P, \mathcal{V})$. If one needs to determine the multiplicity of an infinity point $\bar{P}=\left(0: a_{1}: a_{2}: a_{3}\right), a_{j} \neq 0$ (for some $j \in\{1,2,3\}$ ), we apply the results to the dehomogenization of $\overline{\mathcal{P}}(\bar{t})$ w.r.t. the $j$-component (see [23]).

For this purpose, in the following, $\operatorname{deg}\left(\psi_{\mathcal{P}}\right)$ denotes the degree of the rational map $\psi_{\mathcal{P}}: \mathbb{K}^{2} \longrightarrow \mathcal{V} \subset \mathbb{K}^{3} ; \bar{t} \longmapsto \mathcal{P}(\bar{t})$ induced by the rational parametrization $\mathcal{P}(\bar{t})$. We recall that the birationality of $\psi_{\mathcal{P}}$, i.e. the properness of $\mathcal{P}(\bar{t})$, is characterized by $\operatorname{deg}\left(\psi_{\mathcal{P}}\right)=1$ (see [13] and [27]).

Also, for a rational function $\chi$, we denote by numer $(\chi)$ the numerator of $\chi$ when expressed in reduced form. By $\operatorname{pp}_{\left\{v_{1}, \ldots, v_{n}\right\}}(f)$ and $\operatorname{Content}_{\left\{v_{1}, \ldots, v_{n}\right\}}(f)$, where 
$f \in \mathbb{K}\left[x_{1}, \ldots, x_{m}\right]\left[v_{1}, \ldots, v_{n}\right]$, we denote the primitive part and the content w.r.t. $\left\{v_{1}, \ldots, v_{n}\right\}$ of $f$, respectively. Furthermore, $\operatorname{lc}_{\left\{v_{1}, \ldots, v_{n}\right\}}(f)$ represents the leading coefficient w.r.t $\left\{v_{1}, \ldots, v_{n}\right\}$ of $f$. For $f, g$ polynomials depending on $s$ we denote $\operatorname{Res}_{s}(f, g)$ its resultant.

Theorem 1. ([30]) Consider a rational surface defined by the homogeneous proper parametrization $\overline{\mathcal{P}}\left(t_{0}, t_{1}, t_{2}\right)$ and its corresponding implicit equation $\bar{F}\left(x_{0}, x_{1}, x_{2}, x_{3}\right)=$ 0 . There are three equivalent definitions of a singularity $P$ of order $m$ on the rational surface.

1. All $(m-1)$-th partial derivatives of $\bar{F}\left(x_{0}, x_{1}, x_{2}, x_{3}\right)$ vanish at $P$, and at least one $m$-th partial derivative of $\bar{F}\left(x_{0}, x_{1}, x_{2}, x_{3}\right)$ does not vanish at $P$.

2. The number of intersections (counted properly with multiplicity) of a generic line with the surface at the point $P$ is $m$.

3. The number of parameters $\left(t_{0}, t_{1}, t_{2}\right)$ (counted properly with multiplicity) that correspond to the point $P$ is $m$.

Theorem 2. ([23]) It holds that

$$
\operatorname{mult}(P, \mathcal{V})=\operatorname{deg}(\mathcal{V})-\frac{\operatorname{deg}\left(\psi_{\mathcal{R}_{P}}\right)}{\operatorname{deg}\left(\psi_{\mathcal{P}}\right)},
$$

where $\mathcal{R}_{P}(\bar{t})=\left(\left(p_{2}(\bar{t})-a_{2}\right) /\left(p_{1}(\bar{t})-a_{1}\right),\left(p_{3}(\bar{t})-a_{3}\right) /\left(p_{1}(\bar{t})-a_{1}\right)\right)$. In addition, $\operatorname{deg}(\mathcal{V})=\operatorname{deg}(\overline{\mathcal{V}})=\frac{\operatorname{deg}\left(\psi_{\mathcal{R}_{Q}}\right)}{\operatorname{deg}\left(\psi_{\mathcal{P}}\right)}$, where $Q \notin \mathcal{V}$.

In the following we recall how to compute $\operatorname{deg}\left(\psi_{\mathcal{P}}\right)$ and $\operatorname{deg}\left(\psi_{\mathcal{R}_{P}}\right)$ and we illustrate it with an example. For further details we refer to [20] or [21].

Algorithm 1: Computation of $\operatorname{deg}\left(\psi_{\mathcal{P}}\right)$

[Step 1] For $i=1,2,3$, compute $G_{i}(\bar{t}, \bar{s})=\operatorname{numer}\left(p_{i}(\bar{t})-p_{i}(\bar{s})\right)$, where $\bar{s}=\left(s_{1}, s_{2}\right)$. [Step 2] Determine $R\left(t_{1}, \bar{s}, Z\right)=\operatorname{Res}_{t_{2}}\left(G_{1}, G_{2}+Z G_{3}\right)$, where $Z$ is a new variable.

[Step 3] Compute $\left.S\left(t_{1}, \bar{s}\right)=\operatorname{pp}_{\bar{s}}\left(\operatorname{Content}_{Z}(R)\right)\right)$.

[Step 4] $\operatorname{deg}\left(\psi_{\mathcal{P}}\right)=\operatorname{deg}_{t_{1}}(S)$.

\section{Algorithm 2: Computation of $\operatorname{deg}\left(\psi_{\mathcal{R}_{P}}\right)$}

[Step 1] Let $\mathcal{R}_{P}(\bar{t}):=\left(r_{1}(\bar{t}), r_{2}(\bar{t})\right)$.

[Step 2] For $i=1,2$, let $H_{i}\left(\bar{t}, x_{i}\right)=\operatorname{numer}\left(r_{i}(\bar{t})-x_{i}\right) \in \mathbb{K}\left[\bar{t}, x_{i}\right]$.

[Step 3] $R\left(t_{1}, x_{1}, x_{2}\right)=\operatorname{Res}_{t_{2}}\left(H_{1}, H_{2}\right) \in \mathbb{K}\left[t_{1}, x_{1}, x_{2}\right]$

[Step 4] $\operatorname{deg}\left(\psi_{\mathcal{R}_{P}}\right)=\operatorname{deg}_{t_{1}}\left(\operatorname{pp}_{\left\{x_{1}, x_{2}\right\}}(R)\right)$. 
Example 1. Let $\mathcal{V}$ be a ruled surface over the complex field $\mathbb{C}$ defined by the rational parametrization $\mathcal{P}(\bar{t})=\left(p_{1}(\bar{t}), p_{2}(\bar{t}), p_{3}(\bar{t})\right)=$

$$
\left(\frac{t_{2} t_{1}{ }^{3}+2 t_{2} t_{1}+t_{2} t_{1}{ }^{2}+3 t_{2}-2 t_{1}-t_{1}{ }^{2}-3}{t_{1}{ }^{3}}, \frac{t_{1}{ }^{3}+2 t_{1}-5+12 t_{2} t_{1}{ }^{2}+13 t_{2}}{t_{1}{ }^{3}}, t_{2}\right) .
$$

First, we apply Algorithm 1, and we compute $\operatorname{deg}\left(\psi_{\mathcal{P}}\right)$. For this purpose, we determine the polynomials $G_{i}(\bar{t}, \bar{s})=\operatorname{numer}\left(p_{i}(\bar{t})-p_{i}(\bar{s})\right)$ for $i=1,2,3$. Afterwards, we compute $R\left(t_{1}, \bar{s}, Z\right)=\operatorname{Res}_{t_{2}}\left(G_{1}, G_{2}+Z G_{3}\right)$, and $\left.S\left(t_{1}, \bar{s}\right)=\operatorname{pp}_{\bar{s}}\left(\operatorname{Content}_{Z}(R)\right)\right)=$ $t_{1}-s_{1}$. Thus, we conclude that $\operatorname{deg}\left(\psi_{\mathcal{P}}\right)=\operatorname{deg}_{t_{1}}(S)=1$.

Now, by applying Theorem 2, we compute $\operatorname{deg}(\mathcal{V})$. We consider $Q=(1,2,3) \notin \mathcal{V}$, and we apply Algorithm 2 to compute $\operatorname{deg}\left(\psi_{\mathcal{R}_{Q}}\right)$, where $\mathcal{R}_{Q}(\bar{t})=\left(r_{1}(\bar{t}), r_{2}(\bar{t})\right)=$ $\left(\left(p_{2}(\bar{t})-2\right) /\left(p_{1}(\bar{t})-1\right),\left(p_{3}(\bar{t})-3\right) /\left(p_{1}(\bar{t})-1\right)\right)$. For $i=1,2$, let $H_{i}\left(\bar{t}, x_{i}\right)=$ numer $\left(r_{i}(\bar{t})-x_{i}\right)$. Then, $\operatorname{deg}\left(\psi_{\mathcal{R}_{Q}}\right)=\operatorname{deg}_{t_{1}}\left(\operatorname{pp}_{\left\{x_{1}, x_{2}\right\}}(R)\right)=6$, where $R\left(t_{1}, x_{1}, x_{2}\right)=$ $\operatorname{Res}_{t_{2}}\left(H_{1}, H_{2}\right)$. Hence, $\operatorname{deg}(\mathcal{V})=6$ (note that $\left.\operatorname{deg}\left(\psi_{\mathcal{P}}\right)=1\right)$.

Finally, let us compute the multiplicity of the point $P=(1,1,1)$. For this purpose, we apply Theorem 2, and we have that $\operatorname{mult}(P, \mathcal{V})=\operatorname{deg}(\mathcal{V})-\operatorname{deg}\left(\psi_{\mathcal{R}_{P}}\right)$, where $\mathcal{R}_{P}(\bar{t})=\left(\left(p_{2}(\bar{t})-1\right) /\left(p_{1}(\bar{t})-1\right),\left(p_{3}(\bar{t})-1\right) /\left(p_{1}(\bar{t})-1\right)\right)$. Using Algorithm 2, and reasoning as above, we get that $\operatorname{deg}\left(\psi_{\mathcal{R}_{P}}\right)=3$, and hence $\operatorname{mult}(P, \mathcal{V})=$ $\operatorname{deg}(\mathcal{V})-\operatorname{deg}\left(\psi_{\mathcal{R}_{P}}\right)=6-3=3$ (note that $\left.\operatorname{deg}\left(\psi_{\mathcal{P}}\right)=1\right)$.

\subsection{Preliminaries on Plane Curves: Computation of Singularities}

In this subsection, we deal with the problem of the computation of singularities (as well as their corresponding multiplicities) of a given parametric rational plane curve. For this purpose, it is introduced the T-function, that is a polynomial which is obtained from the computation of a univariate resultant and whose factorization provides the fibre functions of the different ordinary singularities as well as their corresponding multiplicities. From this result, one may easily detect and classify the singularities of the curve (see e.g. [2], [3], [4], [7] or [19]). In the following, we summarize and introduce these notions and results.

Let $\mathcal{C}$ be a rational plane curve defined by the parametrization

$$
\mathcal{P}\left(t_{1}\right)=\left(\frac{q_{1}\left(t_{1}\right)}{q\left(t_{1}\right)}, \frac{q_{2}\left(t_{1}\right)}{q\left(t_{1}\right)}\right) \in \mathbb{K}\left(t_{1}\right)^{2},
$$

where $\operatorname{gcd}\left(q_{1}, q_{2}, q\right)=1$. We assume w.l.o.g. that $\operatorname{deg}\left(q_{i}\right)=\operatorname{deg}(q)=d, i=1,2$ (otherwise, we apply a linear change of variables). The corresponding projective curve $\overline{\mathcal{C}}$ is defined by the parametrization

$$
\overline{\mathcal{P}}\left(t_{1}\right)=\left(q\left(t_{1}\right), q_{1}\left(t_{1}\right), q_{2}\left(t_{1}\right)\right) \in \mathbb{P}^{2}\left(\mathbb{K}\left(t_{1}\right)\right) .
$$


Similarly as in the case of surfaces, associated with $\mathcal{P}\left(t_{1}\right)$, we consider the induced rational map $\psi_{\mathcal{P}}: \mathbb{K} \longrightarrow \mathcal{C} \subset \mathbb{K}^{2} ; t_{1} \longmapsto \mathcal{P}\left(t_{1}\right)$, and $\operatorname{deg}\left(\psi_{\mathcal{P}}\right)$ denotes the degree of the rational map $\psi_{\mathcal{P}}$. We recall that the degree of a rational map can be seen as the cardinality of the fibre of a generic element (see Theorem 7, pp. 76 in [27]). We denote by $\mathcal{F}_{\mathcal{P}}(P)$ the fibre of a point $P \in \mathcal{C}$ via the parametrization $\mathcal{P}$; that is $\mathcal{F}_{\mathcal{P}}(P)=\mathcal{P}^{-1}(P)=\left\{t_{1} \in \mathbb{K} \mid \mathcal{P}\left(t_{1}\right)=P\right\}$.

It is well known that almost all points of $\mathcal{C}$ (except at most a finite number of points) are generated via $\mathcal{P}\left(t_{1}\right)$ by the same number of parameter values, and this number is the degree of $\psi_{\mathcal{P}}$ (see Subsection 2.2. in [25]). Intuitively speaking, the degree of $\psi_{\mathcal{P}}$ measures the number of times that $\mathcal{P}\left(t_{1}\right)$ traces the curve when the parameter takes values in $\mathbb{K}$. Taking into account this intuitive notion, in Chapter 4 in [25], it is proved that $\operatorname{deg}\left(\psi_{\mathcal{P}}\right)=\operatorname{deg}_{t_{1}}(G)$, where $G\left(t_{1}, s_{1}\right)=\operatorname{gcd}\left(G_{1}\left(t_{1}, s_{1}\right), G_{2}\left(t_{1}, s_{1}\right), G_{3}\left(t_{1}, s_{1}\right)\right)$, and

$G_{i}\left(t_{1}, s_{1}\right)=q_{i}\left(s_{1}\right) q\left(t_{1}\right)-q\left(s_{1}\right) q_{i}\left(t_{1}\right), i=1,2, \quad G_{3}\left(t_{1}, s_{1}\right)=q_{1}\left(s_{1}\right) q_{2}\left(t_{1}\right)-q_{2}\left(s_{1}\right) q_{1}\left(t_{1}\right)$.

The cardinality of the fibre is the same for almost all points on $\mathcal{C}$; that is, all but finitely many points in $\mathcal{C}$ are generated, via $\mathcal{P}\left(t_{1}\right)$, by exactly $\operatorname{deg}_{t_{1}}(G)$ parameter values. Nevertheless, for finitely many exceptions, the cardinality may vary. This motivates the following definition.

Definition 1. Let $P \in \mathbb{P}^{2}(\mathbb{K})$, and the projective parametrization $\overline{\mathcal{P}}\left(t_{1}\right) \in \mathbb{P}^{2}\left(\mathbb{K}\left(t_{1}\right)\right)$. We define the fibre function of $P$ via $\overline{\mathcal{P}}\left(t_{1}\right)$ as $H_{P}\left(t_{1}\right):=\operatorname{gcd}\left(G_{1}, G_{2}, G_{3}\right)$.

Note that the roots of $H_{P}$ determine the fibre of $P$. In addition, we observe that the above expression for $H_{P}$ may be simplified (see Remark 2 in [2]). More precisely, if $P$ is an affine point then $H_{P}\left(t_{1}\right)=\operatorname{gcd}\left(G_{1}, G_{2}\right)$. If $P$ is a point at infinity then $H_{P}\left(t_{1}\right)=\operatorname{gcd}\left(p, G_{3}\right)$.

Throughout this paper we assume that $\mathcal{P}$ is a proper parametrization; otherwise, we obtain a proper one by reparametrizing $\mathcal{P}$ (see e.g. [18]). This means that $\operatorname{deg}\left(\psi_{\mathcal{P}}\right)=1$ and, so, the cardinality of the fibre is 1 for almost every point of the curve. However, this cardinality may be different for finitely many points. In fact, the fibre of a singular point is greater than 1 (see e.g. [19]). On the other hand, the cardinality of the fibre may be less than 1 if we consider the limit point of the parametrization. This special point has to be treated separately. The limit point of the parametrization $\overline{\mathcal{P}}\left(t_{1}\right)$ is defined as $P_{L}=\lim _{t_{1} \rightarrow \infty} \overline{\mathcal{P}}\left(t_{1}\right) / t_{1}^{d}=\left(a_{d}: b_{d}: c_{d}\right) \in \mathbb{P}^{2}(\mathbb{K})$.

Theorem 3. Let $\overline{\mathcal{C}}$ be a rational algebraic curve defined by a proper parametrization $\overline{\mathcal{P}}$. Let $P \neq P_{L}$ be a point of $\overline{\mathcal{C}}$ and let $H_{P}\left(t_{1}\right)$ be its fibre function. Then, $\operatorname{mult}(P, \overline{\mathcal{C}})=$ $\operatorname{deg}\left(H_{P}\left(t_{1}\right)\right)$. In addition, for the limit point it holds that $\operatorname{deg}\left(H_{P_{L}}\left(t_{1}\right)\right) \leq \operatorname{mult}\left(P_{L}, \overline{\mathcal{C}}\right)$.

Remark 1. An alternative formulae for computing the multiplicity of a given point is 
provided in [19]. More precisely, let $\left(a_{1}, a_{2}\right) \in \mathbb{K}^{2}$. Then,

$$
\operatorname{mult}\left(\left(a_{1}, a_{2}\right), \mathcal{C}\right)=\operatorname{deg}(\mathcal{C})-\frac{\operatorname{deg}\left(\frac{q_{2}\left(t_{1}\right)-a_{2} q\left(t_{1}\right)}{q_{1}\left(t_{1}\right)-a_{1} q\left(t_{1}\right)}\right)}{\operatorname{deg}\left(\psi_{\mathcal{P}}\right)} .
$$

Note that mult $\left(\left(a_{1}: a_{2}: 1\right), \overline{\mathcal{C}}\right)=\operatorname{mult}\left(\left(a_{1}, a_{2}\right), \mathcal{C}\right)$. In addition, one should note that $\operatorname{mult}\left(P_{L}, \overline{\mathcal{C}}\right)$ has necessarily to be computed using the above formulae. Furthermore, if one needs to determine the multiplicity of an infinity point $\left(0: a_{1}: a_{2}\right), a_{j} \neq 0$ (for some $j \in\{1,2\})$, we apply the multiplicity formula using the dehomogenization of $\overline{\mathcal{P}}\left(t_{1}\right)$ w.r.t. the $j$-component (see [19]).

In [2] (see also [3], [4] and [7]), a method for computing the singularities of a rational algebraic curve from its parametric representation is proposed. The method is based on the construction and factorization of a polynomial called the T-function, which may be defined as

$$
T\left(t_{1}\right)=\frac{R_{12}\left(t_{1}\right)}{q\left(t_{1}\right)^{\lambda_{12}-1}}
$$

where $\bar{G}_{i}\left(t_{1}, s_{1}\right)=G_{i}\left(t_{1}, s_{1}\right) /\left(t_{1}-s_{1}\right)(i=1,2), \lambda_{12}=\min \left\{\operatorname{deg}_{t_{1}}\left(G_{1}\right), \operatorname{deg}_{t_{1}}\left(G_{2}\right)\right\}$, and $R_{12}\left(t_{1}\right)=\operatorname{Res}_{s_{1}}\left(\bar{G}_{1}\left(t_{1}, s_{1}\right), \bar{G}_{2}\left(t_{1}, s_{1}\right)\right)$.

The T-function provides essential information about the singularities of the given curve $\overline{\mathcal{C}}$. More precisely, in Theorem 4 it is proved that the factorization of the Tfunction gives the fibre functions of the singularities of $\overline{\mathcal{C}}$. In order to state this theorem, we assume that $\overline{\mathcal{C}}$ has only ordinary singularities. Otherwise, we apply quadratic transformations (blow-ups) for birationally transforming $\overline{\mathcal{C}}$ into a curve with only ordinary singularities (see Chapter 2 in [25]). For such a curve the following theorem holds (see [2], [3] and [4]).

Theorem 4. Let $\overline{\mathcal{C}}$ be a rational algebraic plane curve defined by a parametrization $\overline{\mathcal{P}}\left(t_{1}\right)$. Let $P_{1}, \ldots, P_{n}$ be the ordinary singular points of $\overline{\mathcal{C}}$, with multiplicities $m_{1}, \ldots, m_{n}$ respectively. Then, up to constants in $\mathbb{K} \backslash\{0\}$, it holds that

$$
T\left(t_{1}\right)=\prod_{i=1}^{n} H_{P_{i}}\left(t_{1}\right)^{m_{i}-1}, \quad \operatorname{gcd}\left(H_{P_{i}}, H_{P_{j}}\right)=1 .
$$

Remark 2. Observe that if $P_{i} \neq P_{L}$ then $\operatorname{deg}\left(H_{P_{i}}\left(t_{1}\right)\right)=m_{i}, i=1, \ldots, n$. Otherwise, $\operatorname{deg}\left(H_{P_{L}}\left(t_{1}\right)\right) \leq \operatorname{mult}\left(P_{L}, \overline{\mathcal{C}}\right)$ (see Theorem 3). For this case $\operatorname{mult}\left(P_{L}, \overline{\mathcal{C}}\right)$ has be computed using the formulae introduced in Remark 1.

The above theorem can be stated for the case of a given curve with non-ordinary singularities (see [4]). More precisely, if $P_{1}, \ldots, P_{\ell} \in \mathbb{P}^{2}(\mathbb{K})$ are the ordinary singular points of $\overline{\mathcal{C}}$, with multiplicities $m_{1}, \ldots, m_{\ell}$, respectively, and $P_{\ell+1}, \ldots, P_{k} \in \mathbb{P}^{2}(\mathbb{K})$ are 
the non-ordinary singular points of $\overline{\mathcal{C}}$, with multiplicities $m_{\ell+1}, \ldots, m_{k}$, respectively then, up to constants in $\mathbb{K} \backslash\{0\}$,

$$
T\left(t_{1}\right)=\prod_{i=1}^{\ell} H_{P_{i}}\left(t_{1}\right)^{m_{i}-1} \prod_{i=\ell+1}^{k} h_{i 1}\left(t_{1}\right)^{r_{i 1}} \cdots h_{i m_{i}}\left(t_{1}\right)^{r_{i m_{i}}},
$$

where $r_{i j} \geq m_{i}-1 \in \mathbb{N}, H_{P_{i}}\left(t_{1}\right)=h_{i 1}\left(t_{1}\right) \cdots h_{i m_{i}}\left(t_{1}\right), \operatorname{gcd}\left(h_{i j}, h_{i k}\right)=1$, for $i=\ell+$ $1, \ldots, k$. For further details on the interpretation of this polynomial see [4].

Finally, we note that, we have been able to determine the singularities of $\overline{\mathcal{C}}$ and its corresponding multiplicities, by computing the factors of the polynomial $T\left(t_{1}\right)$. However, in general, one needs to introduce algebraic numbers during the computations. In the following, we present a method that allows us to determine the factors of the polynomial $T\left(t_{1}\right)$ (and thus, the singularities of a curve) without directly using algebraic numbers. For this purpose, we introduce the notion of family of conjugate parametric points (see [19]). The idea is to collect points whose coordinates depend algebraically on all the conjugate roots of the same irreducible polynomial $m\left(t_{1}\right)$.

Definition 2. Let $\mathcal{G}=\left\{\left(q(\alpha): q_{1}(\alpha): q_{2}(\alpha)\right) \mid m(\alpha)=0\right\} \subset \mathbb{P}^{2}(\mathbb{K})$. The set $\mathcal{G}$ is called a family of conjugate parametric points over $\mathbb{K}$ if the following conditions are satisfied: (1.) $q_{1}, q_{2}, q, m \in \mathbb{K}\left[t_{1}\right]$ and $\operatorname{gcd}\left(q_{1}, q_{2}, q\right)=1$, (2.) $m$ is irreducible and (3.) $\operatorname{deg}\left(q_{1}\right), \operatorname{deg}\left(q_{2}\right), \operatorname{deg}(q)<\operatorname{deg}(m)$. We denote such a family by $\mathcal{G}=\left\{\overline{\mathcal{P}}\left(t_{1}\right)\right\}_{m\left(t_{1}\right)}$.

Remark 3. The family $\mathcal{G}=\left\{\overline{\mathcal{P}}\left(t_{1}\right)\right\}_{m\left(t_{1}\right)}$ is obtained by computing the remainder of $q_{i}\left(t_{1}\right), i=1,2$ modulo $m\left(t_{1}\right)$, and the remainder of $q\left(t_{1}\right)$ modulo $m\left(t_{1}\right)$. For ease of notation we represent by $q_{i}\left(t_{1}\right), i=1,2$ and $q\left(t_{1}\right)$, respectively, the obtained polynomials.

Using the above definition, in [19], the following theorem is proved

Theorem 5. The singularities of a given curve can be decomposed as a finite union of families of conjugate parametric points over $\mathbb{K}$ such that all points in the same family have the same multiplicity and character.

If some singularities of the given curve are in a family $\mathcal{G}=\left\{\overline{\mathcal{P}}\left(t_{1}\right)\right\}_{m\left(t_{1}\right)}$, then the polynomial $m\left(t_{1}\right)$ will be an irreducible factor of the T-function. In this case, Theorem 6 allows us to determine the singularities provided by $\mathcal{G}$ and their corresponding multiplicities.

Theorem 6. Let $m\left(t_{1}\right)$ be an irreducible polynomial such that $m\left(t_{1}\right)^{k-1}, k \in \mathbb{N}, k \geq 1$, divides $T\left(t_{1}\right)$. Then, the roots of $m\left(t_{1}\right)$ determine the fibre of some singularities of multiplicity $k$ that are defined by a family of conjugate parametric points. The number of singularities in such a family is $\operatorname{deg}(m) / k$. 


\section{Singularities of Ruled Surfaces}

In the following, we consider a ruled surface $\mathcal{V}$ defined over $\mathbb{K}$ by a parametrization of the form introduced in (2); that is,

$$
\mathcal{P}(\bar{t})=\left(p_{1}(\bar{t}), p_{2}(\bar{t}), p_{3}(\bar{t})\right)=\left(\frac{p_{11}\left(t_{1}\right)+t_{2} p_{12}\left(t_{1}\right)}{q\left(t_{1}\right)}, \frac{p_{21}\left(t_{1}\right)+t_{2} p_{22}\left(t_{1}\right)}{q\left(t_{1}\right)}, t_{2}\right) \in \mathbb{K}(\bar{t})^{3} .
$$

We denote $q_{i}(\bar{t})=p_{i 1}\left(t_{1}\right)+t_{2} p_{i 2}\left(t_{1}\right)$, for $i=1,2$. We assume w.l.o.g that $\mathcal{P}$ is proper (see [16]) and $\operatorname{deg}_{t_{1}}\left(q_{i}\right)=\operatorname{deg}(q)=d, i=1,2$ (otherwise, one considers a linear change of variables). The corresponding projective surface $\overline{\mathcal{V}}$ is defined by the projective parametrization

$$
\overline{\mathcal{P}}(\bar{t})=\left(q\left(t_{1}\right), p_{11}\left(t_{1}\right)+t_{2} p_{12}\left(t_{1}\right), p_{21}\left(t_{1}\right)+t_{2} p_{22}\left(t_{1}\right), t_{2} q\left(t_{1}\right)\right) \in \mathbb{P}^{3}(\mathbb{K}(\bar{t})) .
$$

In the following, $\overline{\mathcal{F}}$ denotes the algebraic closure of a field $\mathbb{F}$.

From the above rational parametrization, we introduce an auxiliary parametrization of a plane curve defined over $\overline{\mathbb{K}\left(t_{2}\right)}$. This auxiliary parametrization will play an important role on the computation of the singularities $\overline{\mathcal{V}}$. More precisely, we consider the partial parametrization associated to $\mathcal{P}$,

$$
\mathcal{P}_{t_{2}}^{*}\left(t_{1}\right):=\left(\frac{q_{1}(\bar{t})}{q\left(t_{1}\right)}, \frac{q_{2}(\bar{t})}{q\left(t_{1}\right)}\right)=\left(\frac{p_{11}\left(t_{1}\right)+t_{2} p_{12}\left(t_{1}\right)}{q\left(t_{1}\right)}, \frac{p_{21}\left(t_{1}\right)+t_{2} p_{22}\left(t_{1}\right)}{q\left(t_{1}\right)}\right) \in\left(\mathbb{K}\left(t_{2}\right)\right)\left(t_{1}\right)^{2}
$$

(that is, $\mathcal{P}_{t_{2}}^{*}\left(t_{1}\right)$ is defined over $\left.\mathbb{K}\left(t_{2}\right)\right)$. We denote by $\mathcal{C}_{t_{2}}^{*}$ the curve defined by $\mathcal{P}_{t_{2}}^{*}\left(t_{1}\right)$. We observe that the corresponding projective curve $\overline{\mathcal{C}}_{t_{2}}^{*}$ is defined by the projective parametrization

$$
\overline{\mathcal{P}}_{t_{2}}^{*}\left(t_{1}\right)=\left(q\left(t_{1}\right), p_{11}\left(t_{1}\right)+t_{2} p_{12}\left(t_{1}\right), p_{21}\left(t_{1}\right)+t_{2} p_{22}\left(t_{1}\right)\right) \in \mathbb{P}^{2}\left(\left(\mathbb{K}\left(t_{2}\right)\right)\left(t_{1}\right)\right)
$$

In this section, we present an algorithm that allows us to determine the singularities and their multiplicities of $\overline{\mathcal{V}}$ from the parametrization $\overline{\mathcal{P}}$. In fact, we prove that the singularities of the auxiliary curve, $\overline{\mathcal{C}}_{t_{2}}^{*}$, provide the singularities of the input ruled surface and only a finite set of points have to be additionally analyzed (see Theorem 10). This method, which relies on the computation of the $\mathrm{T}$-function for $\overline{\mathcal{P}}_{t_{2}}^{*}$, provides directly the singular points and their multiplicity except for at most some special points. For these points, the multiplicity has to be computed by applying Theorem 2. The ruled surface is an important surface widely used in computer aided geometric design and geometric modeling and thus, easier methods dealing with this special surfaces are welcome.

We remind that, in order to facilitate the reading of the paper, the proofs of the results obtained in this section are presented in Section 4. 


\section{Properties of the Curve $\mathcal{C}_{t_{2}}^{*}$}

To start with, we first prove two propositions where we show some properties of the curve $\mathcal{C}_{t_{2}}^{*}$ and the parametrization $\mathcal{P}_{t_{2}}^{*}\left(t_{1}\right)$.

Proposition 1. If $\mathcal{P}(\bar{t})$ is proper, then $\mathcal{P}_{t_{2}}^{*}\left(t_{1}\right)$ is proper.

In the following proposition, we prove that the non-ordinary singularities of the curve $\overline{\mathcal{C}}_{t_{2}}^{*}$ are of the form $\left(c: a_{1}+t_{2} b_{1}: a_{2}+t_{2} b_{2}\right) \in \mathbb{P}^{2}\left(\mathbb{K}\left(t_{2}\right)\right), a_{i}, b_{i}, c \in \mathbb{K}$. For this purpose, for $f$ a polynomial depending on a variable $v$ we denote $f^{\prime}(v)$ its derivative.

Proposition 2. Let $P=\overline{\mathcal{P}}_{t_{2}}^{*}(s) \in \mathbb{P}^{2}\left(\overline{\mathbb{K}\left(t_{2}\right)}\right)$. If $P$ is an non-ordinary singularity of $\overline{\mathcal{C}}_{t_{2}}^{*}$, then $s \in \mathbb{K}$. In this case, $P=\left(c: a_{1}+t_{2} b_{1}: a_{2}+t_{2} b_{2}\right) \in \mathbb{P}^{2}\left(\mathbb{K}\left(t_{2}\right)\right), a_{i}, b_{i}, c_{i} \in \mathbb{K}$.

\section{Affine Singularities of $\mathcal{V}$}

In Theorem 7, we will show that $\left(a_{1}, a_{2}\right) \in{\overline{\mathbb{K}}\left(t_{2}\right)}^{2}$ is an affine singularity of multiplicity $m$ of $\mathcal{C}_{t_{2}}^{*}$ if and only if $\left(a_{1}, a_{2}, t_{2}\right) \in{\overline{\mathbb{K}}\left(t_{2}\right)}^{3}$ a singularity of multiplicity $m$ of $\mathcal{V}$. For this purpose, we first prove two different lemmas. In the first lemma, we study some particular values of the parameter $t_{2}=\beta \in \mathbb{K}$ that provide some special singularities on the surface $\overline{\mathcal{V}}$ that are of the form $\overline{\mathcal{P}}\left(t_{1}, \beta\right)$. In Lemma 2, we prove that the singularities of $\mathcal{C}_{t_{2}}^{*}$ are preserved when $t_{2}$ takes particular values.

In order to prove these lemmas, we first apply Theorem 2 for the parametrization $\overline{\mathcal{P}}_{t_{2}}^{*}$ of the curve $\overline{\mathcal{C}}_{t_{2}}^{*}$. More precisely, let $P_{1}, \ldots, P_{\ell} \in \mathbb{P}^{2}\left(\overline{\mathbb{K}\left(t_{2}\right)}\right)$ be the ordinary singular points of $\overline{\mathcal{C}}_{t_{2}}^{*}$, with multiplicities $m_{1}, \ldots, m_{\ell}$ respectively, and $P_{\ell+1}, \ldots, P_{k} \in \mathbb{P}^{2}\left(\overline{\mathbb{K}\left(t_{2}\right)}\right)$ be the non-ordinary singular points of $\overline{\mathcal{C}}_{t_{2}}^{*}$, with multiplicities $m_{\ell+1}, \ldots, m_{k}$ respectively (note that from Proposition 2, we get that the non-ordinary singular points are of the form $\left.P_{i}=\overline{\mathcal{P}}_{t_{2}}^{*}(\alpha)=\left(c_{i}: a_{i 1}+b_{i 1} t_{2}: a_{i 2}+b_{i 2} t_{2}\right), \alpha, a_{i j}, b_{i j}, c_{i} \in \mathbb{K}\right)$. Then, from Theorem 4 , we get that

$$
T\left(t_{1}, t_{2}\right)=H\left(t_{2}\right) \prod_{i=1}^{\ell} H_{P_{i}}\left(t_{1}, t_{2}\right)^{m_{i}-1} \prod_{i=\ell+1}^{k} h_{i 1}\left(t_{1}\right)^{r_{i 1}} \cdots h_{i m_{i}}\left(t_{1}\right)^{r_{i m_{i}}},
$$

where $r_{i j} \geq m_{i}-1 \in \mathbb{N}, H_{P_{i}}\left(t_{1}\right)=h_{i 1}\left(t_{1}\right) \cdots h_{i m_{i}}\left(t_{1}\right), \operatorname{gcd}\left(h_{i j}, h_{i k}\right)=1$, for $i=\ell+$ $1, \ldots, k$ (this polynomial can be computed using formula in (4)).

We observe that in equality (5) a new polynomial, $H\left(t_{2}\right) \in \mathbb{K}\left[t_{2}\right]$, appears. We note that, in Theorem 4, the polynomials considered to compute the $\mathrm{T}$-function depend only on the variable $t_{1}$, and the $\mathrm{T}$-function obtained, $T\left(t_{1}\right) \in \mathbb{K}\left[t_{1}\right]$, is unique up to constants in $\mathbb{K} \backslash\{0\}$. In this case, the polynomials considered to compute the $\mathrm{T}$-function depend on the variables $t_{1}, t_{2}$ (although we define as the main variable, the variable $t_{1}$ ). Thus, in this case, the $\mathrm{T}$-function obtained, $T\left(t_{1}, t_{2}\right) \in\left(\mathbb{K}\left[t_{2}\right]\right)\left[t_{1}\right]$, is unique up to constants in $\mathbb{K}\left[t_{2}\right] \backslash\{0\}$. 
Lemma 1. It holds that $H(\beta)=0, \beta \in \mathbb{K}$, if and only if one of the following statements hold:

1. $\mathcal{P}\left(t_{1}, \beta\right) \in \mathbb{K}^{3}$. In this case, $\operatorname{mult}(P, \mathcal{V})=\operatorname{deg}(\mathcal{V})$, where $P:=\mathcal{P}(t, \beta)$.

2. $\mathcal{P}\left(t_{1}, \beta\right)=\left(a_{1}, a_{2}, \beta\right) \in \mathbb{K}\left(t_{1}\right)^{3} \backslash \mathbb{K}^{3}, a_{i} \in \mathbb{K}$, for some $i=1,2$. In this case, $\operatorname{mult}(P, \mathcal{V})=\operatorname{deg}(\mathcal{V})-\operatorname{deg}\left(q / m_{i}\right)$, where $P:=\mathcal{P}(t, \beta)$ and $q_{i}(\bar{t})-a_{i} q\left(t_{1}\right)=$ $\left(t_{2}-\beta\right) m_{i}\left(t_{1}\right)$ for some $i=1,2$.

3. (a) There exists $\alpha \in \mathbb{K}$ such that $q_{i}(\alpha, \beta)=q(\beta)=0, i=1,2$ (i.e. $\alpha$ is a base point of $\left.\mathcal{P}\left(t_{1}, \beta\right)\right)$ or

(b) $\mathcal{P}\left(t_{1}, \beta\right)$ is not proper. In this case, $\operatorname{mult}(P, \mathcal{V})=\operatorname{deg}\left(\psi_{\mathcal{P}\left(t_{1}, \beta\right)}\right)$, where $P:=$ $\mathcal{P}(t, \beta)$.

In the following, we denote by $\mathcal{C}_{\beta}^{*}$ the plane curve obtained from $\mathcal{C}_{t_{2}}^{*}$ when $t_{2}=\beta \in \mathbb{K}$. We assume that $\beta \in \mathbb{K}$ is such that $H(\beta) \neq 0$ (i.e. we are not in the conditions of Lemma 1 ). Thus, $\mathcal{P}_{\beta}^{*}\left(t_{1}\right)$ is a proper parametrization (see statement $3(\mathrm{~b})$ of Lemma 1 ). The corresponding projective curve $\overline{\mathcal{C}}_{\beta}^{*}$ is defined by the projective parametrization

$$
\overline{\mathcal{P}}_{\beta}^{*}\left(t_{1}\right)=\left(q\left(t_{1}\right), p_{11}\left(t_{1}\right)+\beta p_{12}\left(t_{1}\right), p_{21}\left(t_{1}\right)+\beta p_{22}\left(t_{1}\right)\right) \in \mathbb{P}^{2}\left(\mathbb{K}\left(t_{1}\right)\right) .
$$

We apply Theorem 2 for the parametrization $\overline{\mathcal{P}}_{\beta}^{*}$ of the curve $\overline{\mathcal{C}}_{\beta}^{*}$. More precisely, let $Q_{1}, \ldots, Q_{u} \in \mathbb{P}^{2}(\mathbb{K})$ be the ordinary singular points of $\overline{\mathcal{C}}_{\beta}^{*}$, with multiplicities $n_{1}, \ldots, n_{u}$ respectively, and $Q_{u+1}, \ldots, Q_{v} \in \mathbb{P}^{2}(\mathbb{K})$ be the non-ordinary singular points of $\overline{\mathcal{C}}_{\beta}^{*}$, with multiplicities $n_{u+1}, \ldots, n_{v}$ respectively. Then, from Theorem 4 , we get that, up to constants in $\mathbb{K} \backslash\{0\}$,

$$
T_{\beta}\left(t_{1}\right)=\prod_{i=1}^{u} H_{Q_{i}}\left(t_{1}\right)^{n_{i}-1} \prod_{i=u+1}^{v} g_{i 1}\left(t_{1}\right)^{s_{i 1}} \cdots g_{i n_{i}}\left(t_{1}\right)^{s_{i n_{i}}},
$$

where $s_{i j} \geq n_{i}-1 \in \mathbb{N}, H_{Q_{i}}\left(t_{1}\right)=g_{i 1}\left(t_{1}\right) \cdots g_{i n_{i}}\left(t_{1}\right), \operatorname{gcd}\left(g_{i j}, g_{i k}\right)=1$, for $i=u+1, \ldots, v$ (this polynomial can be computed using formula in (4)).

Under these conditions, the following lemma holds.

Lemma 2. Let $\beta \in \mathbb{K}$ be such that $H(\beta) \neq 0$. It holds that, up to constants in $\mathbb{K} \backslash\{0\}$, $T\left(t_{1}, \beta\right)=T_{\beta}\left(t_{1}\right)$. In addition, $Q \in \mathbb{P}^{2}(\mathbb{K})$ is a singularity of $\overline{\mathcal{C}}_{\beta}^{*}$ of multiplicity $m$ if and only if $Q=P(\beta)$, where $P \in \mathbb{P}^{2}\left(\overline{\mathbb{K}\left(t_{2}\right)}\right)$ is a singularity of $\overline{\mathcal{C}}_{t_{2}}^{*}$ of multiplicity $m$.

Using Lemmas 1 and 2, in the following theorem we prove that $\left(a_{1}, a_{2}\right) \in{\overline{\mathbb{K}}\left(t_{2}\right)}^{2}$ is an affine singularity of multiplicity $m$ of $\mathcal{C}_{t_{2}}^{*}$ if and only if $\left(a_{1}, a_{2}, t_{2}\right) \in{\overline{\mathbb{K}}\left(t_{2}\right)}^{3}$ a singularity of multiplicity $m$ of $\mathcal{V}$. 
Theorem 7. The following statements hold:

1. Let $\left(a_{1}, a_{2}\right) \in{\overline{\mathbb{K}}\left(t_{2}\right)}^{2}$ be a singularity of $\mathcal{C}_{t_{2}}^{*}$ of multiplicity $m$. Then, $\left(a_{1}, a_{2}, t_{2}\right) \in$ ${\overline{\mathbb{K}}\left(t_{2}\right)}^{3}$ a singularity of $\mathcal{V}$ of multiplicity $m$.

2. Let $\left(\alpha_{1}, \alpha_{2}, \alpha_{3}\right) \in \mathbb{K}^{3}$ be a singularity of $\mathcal{V}$ of multiplicity $m$ such that $H\left(\alpha_{3}\right) \neq$ 0. Then, $\alpha_{i}=a_{i}\left(\alpha_{3}\right), i=1,2$, where $\left(a_{1}, a_{2}\right) \in{\overline{\mathbb{K}\left(t_{2}\right)}}^{2}$ a singularity of $\mathcal{C}_{t_{2}}^{*}$ of multiplicity $m$

\section{Singularities at the Infinity}

In the following, we deal with the computation of the infinity points that are singularities of the surface $\overline{\mathcal{V}}$ defined by the projective parametrization $\overline{\mathcal{P}}(\bar{t})=$ $\left(q\left(t_{1}\right), q_{1}(\bar{t}), q_{2}(\bar{t}), t_{2} q\left(t_{1}\right)\right) \in \mathbb{P}^{3}(\mathbb{K}(\bar{t}))$.

We show that an infinity singularity of $\overline{\mathcal{V}}$, in general, has a multiplicity greater or equal than the corresponding infinity point of the curve $\overline{\mathcal{C}}_{t_{2}}^{*}$ defined by the parametrization $\overline{\mathcal{P}}_{t_{2}}^{*}\left(t_{1}\right)=\left(q\left(t_{1}\right), q_{1}(\bar{t}), q_{2}(\bar{t})\right) \in \mathbb{P}^{2}\left(\left(\mathbb{K}\left(t_{2}\right)\right)\left(t_{1}\right)\right)$. Hence, in order to study these infinity points, one has to determine the multiplicity of the points $P:=\overline{\mathcal{P}}\left(\alpha, t_{2}\right) \in$ $\mathbb{P}^{3}\left(\mathbb{K}\left(t_{2}\right)\right)$, where $q(\alpha)=0$, using Theorem 2. Observe that since one has an infinity point of the form $P=\left(0: a_{1}: a_{2}: 0\right), a_{j} \neq 0$ (for some $\left.j \in\{1,2\}\right)$, we apply the multiplicity formula using the dehomogenization of $\overline{\mathcal{P}}(\bar{t})$ w.r.t. the $j$-component.

Theorem 8. Let $\left(0: a_{1}: a_{2}\right) \in \mathbb{P}^{2}\left(\overline{\mathbb{K}\left(t_{2}\right)}\right)$ be a singularity of $\overline{\mathcal{C}}_{t_{2}}^{*}$ of multiplicity $m$. Then, $P:=\left(0: a_{1}: a_{2}: 0\right) \in \mathbb{P}^{3}\left(\overline{\mathbb{K}\left(t_{2}\right)}\right)$ is a singularity of $\overline{\mathcal{V}}$ and

$$
\operatorname{mult}(P, \overline{\mathcal{V}})=\operatorname{deg}(\mathcal{V})-\operatorname{deg}_{t_{1}}\left(\frac{a_{2} q_{1}(\bar{t})-a_{1} q_{2}(\bar{t})}{q\left(t_{1}\right)}\right) \geq m
$$

Remark 4. Theorem 8 implies that $\left(0: a_{1}: a_{2}: 0\right) \in \mathbb{P}^{3}\left(\overline{\mathbb{K}\left(t_{2}\right)}\right)$ could be a singularity of $\overline{\mathcal{V}}$ and the corresponding point $\left(0: a_{1}: a_{2}\right) \in \mathbb{P}^{2}\left(\overline{\mathbb{K}\left(t_{2}\right)}\right)$ be a simple point of $\overline{\mathcal{C}}_{t_{2}}^{*}$.

\section{Analysis of the Limit Point}

We finally analyze the limit point $P_{L}$ of the parametrization $\overline{\mathcal{P}}_{t_{2}}^{*}\left(t_{1}\right)$. Taking into account Theorems 3 and 4, and Remarks 1 and 2, we observe that $P_{L}$ can be a singular point of $\overline{\mathcal{C}}_{t_{2}}^{*}$ (affine or at the infinity) and $\operatorname{deg}\left(H_{P_{L}}\right)=0$ (note that $\operatorname{deg}\left(H_{P_{L}}\left(t_{1}\right)\right) \leq$ $\left.\operatorname{mult}\left(P_{L}, \overline{\mathcal{C}}_{t_{2}}^{*}\right)\right)$. Thus, this factor could not appear in the polynomial $T(\bar{t})$. In addition, we observe that since $\operatorname{deg}_{t_{1}}\left(q_{i}\right)=\operatorname{deg}(q)=d, i=1,2$, we have that

$$
P_{L}=\lim _{t_{1} \rightarrow \infty} \overline{\mathcal{P}}_{t_{2}}^{*}\left(t_{1}\right) / t_{1}^{d}=\left(a_{d}: b_{d, 1}+t_{2} c_{d, 1}: b_{d, 2}+t_{2} c_{d, 2}\right) \in \mathbb{P}^{2}\left(\mathbb{K}\left(t_{2}\right)\right),
$$

where $a_{d}:=\mathrm{lc}_{t_{1}}(q) \in \mathbb{K} \backslash\{0\}, b_{d, i}:=\mathrm{lc}_{t_{1}}\left(p_{i 1}\right) \in \mathbb{K}, c_{d, i}:=\mathrm{lc}_{t_{1}}\left(p_{i 2}\right) \in \mathbb{K}$, for $i=1,2$. Observe that $H_{P_{L}}\left(t_{1}\right) \in \mathbb{K}\left[t_{1}\right]$ (i.e. this polynomial does not depend on the variable $t_{2}$ ). 
Under these conditions, we observe that the corresponding point on the surface $\overline{\mathcal{V}}$ is $\left(a_{d}: b_{d, 1}+t_{2} c_{d, 1}: b_{d, 2}+t_{2} c_{d, 2}: a_{d} t_{2}\right)$. From Theorem 2, one has the following theorem.

Theorem 9. Let $P=\left(a_{d}: b_{d, 1}+t c_{d, 1}: b_{d, 2}+t c_{d, 2}: a_{d} t\right) \in \mathbb{P}^{3}(\mathbb{K}(t))$. It holds that $\operatorname{mult}(P, \overline{\mathcal{V}})=\operatorname{deg}(\mathcal{V})-\operatorname{deg}\left(\psi_{\mathcal{R}_{P}}\right)$, where

$$
\mathcal{R}_{P}(\bar{t})=\left(\frac{p_{2}(\bar{t})-\alpha_{2}}{p_{1}(\bar{t})-\alpha_{1}}, \frac{t_{2}-\alpha_{3}}{p_{1}(\bar{t})-\alpha_{1}}\right), \alpha_{i}=\left(b_{d, i}+t c_{d, i}\right) / a_{d}, i=1,2, \quad \alpha_{3}=t .
$$

From the above results, we obtain the following theorem that allow to determine the singularities of $\overline{\mathcal{V}}$ using the T-function of the curve $\overline{\mathcal{C}}_{t_{2}}^{*}$. For this purpose, we use the equality (5) and in particular, we represent the $\mathrm{T}$-function as

$$
T\left(t_{1}, t_{2}\right)=H\left(t_{2}\right) \prod_{i=1}^{\ell} H_{P_{i}}\left(t_{1}, t_{2}\right)^{m_{i}-1} \prod_{i=\ell+1}^{k} h_{i 1}\left(t_{1}\right)^{r_{i 1}} \cdots h_{i m_{i}}\left(t_{1}\right)^{r_{i m_{i}}},
$$

where $r_{i j} \geq m_{i}-1 \in \mathbb{N}, H_{P_{i}}\left(t_{1}\right)=h_{i 1}\left(t_{1}\right) \cdots h_{i m_{i}}\left(t_{1}\right)$, for $i=\ell+1, \ldots, k$ (this polynomial can be computed using formula in (4)).

\section{Main Result}

Theorem 10. (Main Theorem). $P$ is a singularity of $\overline{\mathcal{V}}$ if and only if one of the following statements holds:

1. P belongs to a family $\mathcal{G}_{i}=\{\overline{\mathcal{P}}(\alpha, \beta)\}_{m_{i}(\alpha, \beta)}$, where $m_{i}(\bar{t}) \in \mathbb{K}[\bar{t}] \backslash \mathbb{K}\left[t_{i}\right], i=1,2$, is an irreducible polynomial such that $m_{i}(\bar{t})^{k_{i}-1}, k_{i} \in \mathbb{N}, k_{i} \geq 1$, divides $T(\bar{t})$, for some $i=1, \ldots, \ell$.

2. $P=\overline{\mathcal{P}}\left(\alpha, t_{2}\right)$, where $H_{P_{i}}(\alpha)=0$ for some $i=\ell+1, \ldots, k$.

3. $P=\left(a_{d}: b_{d, 1}+t c_{d, 1}: b_{d, 2}+t c_{d, 2}: a_{d} t\right) \in \mathbb{P}^{3}(\mathbb{K}(t))$, where $a_{d}:=\operatorname{lc}_{t_{1}}(q) \in$ $\mathbb{K} \backslash\{0\}, b_{d, i}:=\mathrm{lc}_{t_{1}}\left(p_{i 1}\right) \in \mathbb{K}, c_{d, i}:=\mathrm{lc}_{t_{1}}\left(p_{i 2}\right) \in \mathbb{K}$, for $i=1,2$.

4. $P=\overline{\mathcal{P}}\left(t_{1}, \beta\right)$, where $H(\beta)=0$.

5. $P=\overline{\mathcal{P}}\left(\alpha, t_{2}\right)$, where $q(\alpha)=0$.

6. $P$ belongs to the missed line of the surface $\overline{\mathcal{V}}$ that is not reached by $\overline{\mathcal{P}}$.

Remark 5. We observe that:

1. Theorem 7 implies that the family $\mathcal{G}_{i}=\{\overline{\mathcal{P}}(\alpha, \beta)\}_{m_{i}(\alpha, \beta)}$ of statement 1 of Theorem 10 is a singularity of multiplicity $k_{i}$ of $\overline{\mathcal{V}}$ (see Theorem 5). In addition, the number of singularities in the family $\mathcal{G}_{i}$ is $\operatorname{deg}_{t_{1}}\left(m_{i}\right) / k_{i}$ (see Theorem 6). Observe that $\operatorname{mult}\left(\mathcal{G}_{i}, \overline{\mathcal{V}}\right)$ is the multiplicity of the corresponding family on the curve $\mathcal{C}_{t_{2}}^{*}$ (see Theorem 7).

2. The multiplicity on $\overline{\mathcal{V}}$ of the points obtained in statement 2 of Theorem 10 can be computed by applying Theorem 2 or determining $\operatorname{deg}\left(H_{P_{i}}\left(t_{1}\right)\right.$ ) (if $P_{i}$ is not the limit point; see Theorems 3 and 7). Note that the non-ordinary singularities of the curve $\overline{\mathcal{C}}_{t_{2}}^{*}$ are included in these set of points (see Proposition 2). 
3. Statement 3 of Theorem 10 corresponds to the limit point of $\overline{\mathcal{P}}_{t_{2}}^{*}$ and the multiplicity can be computed by applying Theorem 9. If this point is reached by the parametrization $\overline{\mathcal{P}}_{t_{2}}^{*}$ then this point appears in statement 2.

4. Statement 4 of Theorem 10 corresponds to the points analyzed in Lemma 1. The multiplicity of these points can be computed by applying Lemma 1 (or equivalently Theorem 2).

5. Statement 5 of Theorem 10 corresponds to the infinity points of the surface $\overline{\mathcal{V}}$ (which are obtained from the roots of the denominator of the parametrization, $\left.q\left(t_{1}\right)\right)$. The multiplicity of these points has to be computed by applying Theorem 2, and Algorithm 2 (see Theorem 8 and Remark 4).

6. In statements 2,4 and 5 of Theorem 10, one also may consider families of conjugate parametric points (see Definition 2). In addition, for these cases, Theorems 5 and 6 also work.

7. From the results of [26], we know that every rational ruled surface can be covered with at most two surface parametrizations. In fact, every rational ruled surface can be parametrized in an standardized way that misses at most one line. This line can be computed by applying Theorem 2.6 in [26].

\subsection{Algorithm and Examples}

Results obtained in this section allow to compute all the singularities of a given ruled surface defined by a standard reduced form parametrization, and their multiplicities without computing the implicit equation and without introducing algebraic numbers. In particular, the ideas described in the theorems obtained above, immediately yield the following algorithm.

\section{Main Algorithm: Parametric-Decomposition-Singularities for Ruled Surfaces.}

Given the surface $\overline{\mathcal{V}}$ defined by the projective proper parametrization

$$
\overline{\mathcal{P}}(\bar{t})=\left(q\left(t_{1}\right), p_{11}\left(t_{1}\right)+t_{2} p_{12}\left(t_{1}\right), p_{21}\left(t_{1}\right)+t_{2} p_{22}\left(t_{1}\right), t_{2} q\left(t_{1}\right)\right) \in \mathbb{P}^{3}(\mathbb{K}(\bar{t})),
$$

the algorithm computes a set, $\mathcal{S}$, containing the decomposition of all the singularities of $\overline{\mathcal{V}}$, and their corresponding multiplicities.

[Step 1] Apply formula in (4) to compute the T-function of the curve $\overline{\mathcal{C}}_{t_{2}}^{*}$ defined by the parametrization

$$
\overline{\mathcal{P}}_{t_{2}}^{*}\left(t_{1}\right)=\left(q\left(t_{1}\right), p_{11}\left(t_{1}\right)+t_{2} p_{12}\left(t_{1}\right), p_{21}\left(t_{1}\right)+t_{2} p_{22}\left(t_{1}\right)\right) \in \mathbb{P}^{2}\left(\left(\mathbb{K}\left(t_{2}\right)\right)\left(t_{1}\right)\right) .
$$

Represents this polynomial as in equality (5). Set $\mathcal{S}=\emptyset$. 
[Step 2] For each irreducible polynomial, $m_{i}(\bar{t})$, such that $m_{i}(\bar{t})^{k_{i}-1}, k_{i} \in \mathbb{N}, k_{i} \geq 1$, divides $T(\bar{t})$, consider the family $\mathcal{G}_{i}=\{\overline{\mathcal{P}}(\bar{t})\}_{m_{i}(\bar{t})}$. Do $\mathcal{S}=\mathcal{S} \cup\left[\mathcal{G}_{i}, k_{i}\right]$ (see statement 1 in Remark 5).

[Step 3] Let $m_{\alpha}$ be the multiplicity of $P_{\alpha}=\overline{\mathcal{P}}\left(\alpha, t_{2}\right)$, where $H_{P_{i}}(\alpha)=0$ for some $i=\ell+1, \ldots, k$ (see statement 2 in Remark 5). Do $\mathcal{S}=\mathcal{S} \cup\left[P_{\alpha}, m_{\alpha}\right]$.

[Step 4] Check whether $P=\left(a_{d}: b_{d, 1}+t c_{d, 1}: b_{d, 2}+t c_{d, 2}: a_{d} t\right)$, where $a_{d}:=\operatorname{lc}_{t_{1}}(q) \in$ $\mathbb{K} \backslash\{0\}, b_{d, i}:=\mathrm{lc}_{t_{1}}\left(p_{i 1}\right) \in \mathbb{K}, c_{d, i}:=\mathrm{lc}_{t_{1}}\left(p_{i 2}\right) \in \mathbb{K}$, for $i=1,2$ appears in Step 3. In the affirmative case, go to Step 5. Otherwise, apply Theorem 9 to check whether $P$ is a singularity (see statement 3 in Remark 5 ). In the affirmative case let $m$ be its multiplicity. Do $\mathcal{S}=\mathcal{S} \cup[P, m]$.

[Step 5] Apply Lemma 1 to compute the multiplicity of $P_{\beta}=\overline{\mathcal{P}}\left(t_{1}, \beta\right)$, where $H(\beta)=0$ (see statement 4 in Remark 5). Let $m_{\beta}$ be this multiplicity. Do $\mathcal{S}=\mathcal{S} \cup\left[P_{\beta}, m_{\beta}\right]$.

[Step 6] Apply Theorem 2 to check whether $Q_{\alpha}=\overline{\mathcal{P}}\left(\alpha, t_{2}\right)$, where $q(\alpha)=0$, is a singularity (see statement 5 in Remark 5 ). In the affirmative case let $m_{\alpha}$ be its multiplicity. Do $\mathcal{S}=\mathcal{S} \cup\left[Q_{\alpha}, m_{\alpha}\right]$.

[Step 7] Compute the missed line, $L$, of the parametrization $\overline{\mathcal{P}}$ by applying Theorem 2.6 in [26]. Afterwards, check whether $L$ is a singularity by applying Theorem 2. In the affirmative case let $m$ be its multiplicity. Do $\mathcal{S}=\mathcal{S} \cup[L, m]$.

[Step 8] Return $\mathcal{S}$.

Remark 6. The implicit equations of the singular curves obtained in the algorithm can be directly computed by applying elimination theory techniques as Gröbner bases, techniques based on the computation of univariate resultants (see e.g. [22], [25]), etc.

Example 2. We consider the surface introduced in Example 1. More precisely, let $\mathcal{V}$ be the ruled surface over the complex field $\mathbb{C}$ defined by the rational parametrization $\mathcal{P}(\bar{t})=\left(p_{1}(\bar{t}), p_{2}(\bar{t}), p_{3}(\bar{t})\right)=$

$$
\begin{gathered}
\left(\frac{q_{1}\left(t_{1}, t_{2}\right)}{q\left(t_{1}\right)}, \frac{q_{2}\left(t_{1}, t_{2}\right)}{q\left(t_{1}\right)}, t_{2}\right)=\left(\frac{p_{11}\left(t_{1}\right)+t_{2} p_{12}\left(t_{1}\right)}{q\left(t_{1}\right)}, \frac{p_{21}\left(t_{1}\right)+t_{2} p_{22}\left(t_{1}\right)}{q\left(t_{1}\right)}, t_{2}\right)= \\
\left(\frac{t_{2} t_{1}{ }^{3}+2 t_{2} t_{1}+t_{2} t_{1}^{2}+3 t_{2}-2 t_{1}-t_{1}^{2}-3}{t_{1}^{3}}, \frac{t_{1}{ }^{3}+2 t_{1}-5+12 t_{2} t_{1}{ }^{2}+13 t_{2}}{t_{1}{ }^{3}}, t_{2}\right) .
\end{gathered}
$$

In Example 1, we obtained that $\operatorname{deg}\left(\psi_{\mathcal{P}}\right)=1$ (i.e. $\mathcal{P}$ is proper), and $\operatorname{deg}(\mathcal{V})=6$. We apply Algorithm Parametric-Decomposition-Singularities for Ruled Surfaces to determine the singularities of $\overline{\mathcal{V}}$. For this purpose, we first apply formula in (4) to compute the $T$-function corresponding to the plane curve $\mathcal{C}_{t_{2}}^{*}$ defined by $\mathcal{P}_{t_{2}}^{*}\left(t_{1}\right):=\left(p_{1}(\bar{t}), p_{2}(\bar{t})\right) \in$ $\left(\mathbb{C}\left[t_{2}\right]\right)\left(t_{1}\right)^{2}$. We apply formula in (4), and we get $T(\bar{t})=$

$$
\left(256+238 t_{2} t_{1}+80 t_{1}-832 t_{2}+1153 t_{2}^{2} t_{1}^{2}-206 t_{2} t_{1}^{2}-598 t_{1} t_{2}^{2}+57 t_{1}^{2}+676 t_{2}^{2}\right)\left(-1+t_{2}\right)^{2} .
$$


Let $H\left(t_{2}\right)=\left(-1+t_{2}\right)^{2}$ and $m(\bar{t}):=$

$$
256+238 t_{2} t_{1}+80 t_{1}-832 t_{2}+1153 t_{2}^{2} t_{1}^{2}-206 t_{2} t_{1}^{2}-598 t_{1} t_{2}^{2}+57 t_{1}^{2}+676 t_{2}^{2} .
$$

In step 2 of the algorithm, we get that the family $\mathcal{G}=\{\overline{\mathcal{P}}(\bar{t})\}_{m(\bar{t})}$ is a singularity of multiplicity $k=2$. In addition, the number of singularities in the family is $\operatorname{deg}_{t_{1}}(m) / k=1$ (see statement 1 in Remark 5). Then, $\mathcal{S}=[\mathcal{G}, 2]$.

In step 4 of the algorithm, we check whether $P=(1: t: 1: t)$ is a singularity of $\overline{\mathcal{V}}$ (see statement 3 in Remark 5). We get that $\operatorname{mult}(P, \mathcal{V})=1$ and thus $P$ is not a singular point.

Now, we apply step 5 of the algorithm and we check whether $P=\mathcal{P}(t, 1)=\left(1,\left(t^{3}+\right.\right.$ $\left.\left.2 t+8+12 t^{2}\right) / t^{3}, 1\right)$ is a singularity (see statement 4 in Remark 5). Observe that $P$ parametrizes the line $x_{1}=x_{3}=1$. For this purpose, we apply Lemma 1 or Theorem 2. Let us apply Theorem 2 and Algorithm 2, and we reason similarly as in Example 1. We have that $\operatorname{mult}(P, \mathcal{V})=\operatorname{deg}(\mathcal{V})-\operatorname{deg}\left(\psi_{\mathcal{R}_{P}}\right)$, where

$$
\begin{gathered}
\mathcal{R}_{P}(\bar{t})=\left(r_{1}(\bar{t}), r_{2}(\bar{t})\right)=\left(\frac{p_{2}(\bar{t})-\left(t^{3}+2 t+8+12 t^{2}\right) / t^{3}}{p_{1}(\bar{t})-1}, \frac{p_{3}(\bar{t})-1}{p_{1}(\bar{t})-1}\right)= \\
\left(\frac{2 t^{3} t_{1}-5 t^{3}+12 t^{3} t_{2} t_{1}^{2}+13 t_{2} t^{3}-2 t_{1}^{3} t-8 t_{1}^{3}-12 t_{1}^{3} t^{2}}{t^{3}\left(t_{1}^{3}+2 t_{1}+t_{1}^{2}+3\right)\left(-1+t_{2}\right)}, \frac{t_{1}^{3}}{t_{1}^{3}+2 t_{1}+t_{1}^{2}+3}\right) .
\end{gathered}
$$

(note that $\operatorname{deg}\left(\psi_{\mathcal{P}}\right)=1$ ). Using Algorithm 2, we compute $\operatorname{deg}\left(\psi_{\mathcal{R}_{P}}\right)$. For $i=1,2$, let $H_{i}\left(\bar{t}, x_{i}\right)=\operatorname{numer}\left(r_{i}(\bar{t})-x_{i}\right)$. Then,

$$
R\left(t_{1}, x_{1}, x_{2}\right)=\operatorname{Res}_{t_{2}}\left(H_{1}, H_{2}\right)=t_{1}^{3}-x_{2} t_{1}^{3}-2 x_{2} t_{1}-x_{2} t_{1}^{2}-3 x_{2} .
$$

Therefore, $\operatorname{deg}\left(\psi_{\mathcal{R}_{P}}\right)=\operatorname{deg}_{t_{1}}\left(\operatorname{pp}_{\left\{x_{1}, x_{2}\right\}}(R)\right)=3$, and hence $\operatorname{mult}\left(P_{1}, \mathcal{V}\right)=\operatorname{deg}(\mathcal{V})-$ $\operatorname{deg}\left(\psi_{\mathcal{R}_{P}}\right)=6-3=3$. Then, $\mathcal{S}=[\mathcal{G}, 2] \cup[P, 3]$.

In step 6 of the algorithm, we check whether $Q=\mathcal{P}(0, t)=(0:-3+3 t:-5+13 t: 0)$ is a singularity (see statement 5 in Remark 5). For this purpose, we apply Theorem 2 and Algorithm 2, and we get that $\operatorname{mult}(Q, \mathcal{V})=3$. Thus $\mathcal{S}=[\mathcal{G}, 2] \cup[P, 3] \cup[Q, 3]$.

Finally, we compute the missed line, L, of the parametrization $\overline{\mathcal{P}}$ by applying Theorem 2.6 in [26], and we get that $L$ is not a singularity. Thus, we return

$$
\mathcal{S}=[\mathcal{G}, 2] \cup[P, 3] \cup[Q, 3] .
$$

Example 3. Let $\mathcal{V}$ be a ruled surface over the complex field $\mathbb{C}$ defined by the rational parametrization $\mathcal{P}(\bar{t})=\left(p_{1}(\bar{t}), p_{2}(\bar{t}), p_{3}(\bar{t})\right) \in \mathbb{C}(\bar{t})^{3}$, where $p_{1}(\bar{t})=-1 / 17 \frac{370-153 t_{1}-68 t_{1}^{2}-85 t_{1}^{3}-17 t_{2} t_{1}^{2}-153 t_{2} t_{1}+1620 t_{2}+136 t_{2} t_{1}^{3}}{t_{1}^{2}\left(1+t_{1}\right)}$, 
$p_{2}(\bar{t})=-1 / 17 \frac{1330+323 t_{1}-238 t_{1}{ }^{2}+85 t_{1}{ }^{3}-17 t_{2} t_{1}{ }^{2}-323 t_{2} t_{1}+3420 t_{2}+306 t_{2} t_{1}{ }^{3}}{t_{1}{ }^{2}\left(1+t_{1}\right)}$,

and $p_{3}(\bar{t})=t_{2}$. Using Algorithms 1 and 2 , one gets that $\operatorname{deg}\left(\psi_{\mathcal{P}}\right)=1$ (i.e. $\mathcal{P}$ is proper), and $\operatorname{deg}(\mathcal{V})=6$. Now, we apply Algorithm Parametric-Decomposition-Singularities for Ruled Surfaces to compute the singularities of $\overline{\mathcal{V}}$. For this purpose, we first apply formula in (4) to determine the $T$-function corresponding to the plane curve $\mathcal{C}_{t_{2}}^{*}$ defined by $\mathcal{P}_{t_{2}}^{*}\left(t_{1}\right):=\left(p_{1}(\bar{t}), p_{2}(\bar{t})\right) \in\left(\mathbb{C}\left[t_{2}\right]\right)\left(t_{1}\right)^{2}$. We get

$$
T(\bar{t})=\left(7 t_{2}+2\right)^{2}\left(t_{1}-5\right)^{2} .
$$

Let $H\left(t_{2}\right)=\left(7 t_{2}+2\right)^{2}$ and $H_{P}\left(t_{1}\right):=\left(t_{1}-5\right)^{2}$.

We apply step 3 of the algorithm and we compute the multiplicity of

$$
P_{1}=\mathcal{P}(5, t)=(424 / 85-581 / 85 t,-254 / 85-1321 / 85 t, t)
$$

(see statement 2 in Remark 5). Observe that $P_{1}$ parametrizes the line $x_{1}-424 / 85+$ $581 / 85 x_{3}=x_{2}+254 / 85+1321 / 85 x_{3}=0$ (apply Remark 6). For this purpose, we apply Theorem 2 and Algorithm 2, and we get that $\operatorname{deg}\left(\psi_{\mathcal{R}_{P}}\right)=4$. Hence $\operatorname{mult}(P, \mathcal{V})=$ $\operatorname{deg}(\mathcal{V})-\operatorname{deg}\left(\psi_{\mathcal{R}_{P}}\right)=6-4=2$. Observe that, this multiplicity can be directly obtained by computing $\operatorname{deg}\left(H_{P}\left(t_{1}\right)\right)=2$ (see statement 2 in Remark 5). Then, $\mathcal{S}=\left[P_{1}, 2\right]$.

In step 4 of the algorithm, we check whether $P=(1: 5-8 t:-5-18 t: t)$ is a singularity of $\overline{\mathcal{V}}$ (see statement 3 in Remark 5). Observe that this point is not obtained in step 3 of the algorithm. We get that $\operatorname{mult}(P, \mathcal{V})=1$ and thus $P$ is not a singular point.

In step 5 of the algorithm, we check whether $P_{2}=\mathcal{P}(t,-2 / 7)=$

$$
\left(\frac{1}{119} \frac{650+765 t+442 t^{2}+867 t^{3}}{t^{2}(1+t)}, \frac{1}{119} \frac{-2470-2907 t+1632 t^{2}+17 t^{3}}{t^{2}(1+t)},-2 / 7\right)
$$

is a singularity (see statement 4 in Remark 5). For this purpose, we apply Lemma 1 (also Theorem 2 can be applied). We get that $\operatorname{mult}\left(P_{2}, \mathcal{V}\right)=3$, and then $\mathcal{S}=$ $\left[P_{1}, 2\right] \cup\left[P_{2}, 3\right]$. Note that $P_{2}$ parametrizes the line $133 x_{1}-974+35 x_{2}=x_{3}+2 / 7=0$ (apply Remark 6).

Now, we apply step 6 of the algorithm to check whether

$$
\begin{gathered}
Q_{1}=\mathcal{P}(0, t)=(0:-185-810 t:-665-1710 t: 0), \text { and } \\
Q_{2}=\mathcal{P}(-1, t)=(0:-135-405 t:-171-855 t: 0)
\end{gathered}
$$

are singularities (see statement 5 in Remark 5). For this purpose, we apply Theorem 2 and Algorithm 2, and we get that mult $\left(Q_{i}, \mathcal{V}\right)=3, i=1,2$. Thus $\mathcal{S}=\left[P_{1}, 2\right] \cup\left[P_{2}, 3\right] \cup$ $\left[Q_{1}, 3\right] \cup\left[Q_{2}, 3\right]$. 
Example 4. In this example, we consider the Example 4.2 of [14]. More precisely, let $\mathcal{V}$ be a ruled surface over the complex field $\mathbb{C}$ defined by the rational parametrization $\mathcal{P}(\bar{t})=\left(p_{1}(\bar{t}), p_{2}(\bar{t}), p_{3}(\bar{t})\right)$, where

$$
\begin{gathered}
p_{1}=\frac{\left(-3+15 t_{1}+3 t_{1}^{4} t_{2}-t_{1}^{3} t_{2}+36 t_{1}^{4}-13 t_{1}^{3}-18 t_{1}^{2}-2 t_{1}^{5} t_{2}+18 t_{1}^{6}-26 t_{1}^{5}\right)\left(t_{1}+1\right)}{t_{1}^{4}\left(t_{1}^{3}-2 t_{1}^{2}+3 t_{1}-1\right)}, \\
p_{2}=\frac{-7 t_{1}^{4}+20 t_{1}^{3}-20 t_{1}^{2}-5 t_{1}+5+t_{1}^{4} t_{2}}{t_{1}^{4}}, \quad p_{3}=t_{2}
\end{gathered}
$$

(we consider a reparametrization of the input parametrization of Example 4.2 of [14] to get an standard reduced form parametrization of $\mathcal{V}$; see (2)). Reasoning as in Example 1, we obtain that $\operatorname{deg}\left(\psi_{\mathcal{P}}\right)=1$ (i.e. $\mathcal{P}$ is proper), and $\operatorname{deg}(\mathcal{V})=8$. We apply Algorithm Parametric-Decomposition-Singularities for Ruled Surfaces to determine the singularities of $\overline{\mathcal{V}}$. For this purpose, we first apply formula in (4) to compute the T-function of the plane curve $\mathcal{C}_{t_{2}}^{*}$ defined by $\mathcal{P}_{t_{2}}^{*}\left(t_{1}\right):=\left(p_{1}(\bar{t}), p_{2}(\bar{t})\right) \in\left(\mathbb{C}\left[t_{2}\right]\right)\left(t_{1}\right)^{2}$. We get that $T(\bar{t})=$$$
-109375 t_{1}^{6}\left(-8+t_{2}\right)^{6}\left(2 t_{1}-1\right)^{2}\left(t_{1}-1\right)^{2}\left(t_{1}^{4}-4 t_{1}^{3}+4 t_{1}^{2}+t_{1}-1\right)^{3}\left(t_{1}^{3}-2 t_{1}^{2}+3 t_{1}-1\right)^{2} .
$$

Let

$H\left(t_{2}\right)=\left(-8+t_{2}\right)^{6}, \quad m\left(t_{1}\right):=t_{1}^{4}-4 t_{1}^{3}+4 t_{1}^{2}+t_{1}-1$, and $H_{P_{1}}\left(t_{1}\right)=\left(2 t_{1}-1\right)\left(t_{1}-1\right)$

(note that $\left.P_{1}=\mathcal{P}(1 / 2, t)=\mathcal{P}(1, t)\right)$. The remainder factors in the polynomial $T$ correspond to the denominator of the parametrization. These factors will provide infinity points which are studied in step 6 of the algorithm.

In step 3 of the algorithm, we get that the family $\mathcal{G}=\{\overline{\mathcal{P}}(\bar{t})\}_{m\left(t_{1}\right)}$ is a singularity of multiplicity $k=4$. In addition, the number of singularities in the family is $\operatorname{deg}_{t_{1}}(m) / k=1$ (see statements 2 and 6 in Remark 5). One may check that $\mathcal{G}$ parametrizes the line defined by $x_{1}-29+x_{3}=x_{2}+2-x_{3}=0$ (see Remark 6 ). Then, $\mathcal{S}=[\mathcal{G}, 4]$

Also in this step, we compute the multiplicity of the point $P_{1}=\mathcal{P}(1 / 2, t)=\mathcal{P}(1, t)=$ $(18, t-7, t)$ (see statement 2 in Remark 5). We apply Theorem 2 , and we get that $\operatorname{mult}\left(P_{1}, \mathcal{V}\right)=3$. Then, $\mathcal{S}=[\mathcal{G}, 4] \cup\left[P_{1}, 3\right]$. Observe that $P_{1}$ parametrizes the line defined by $x_{1}-18=x_{2}+7-x_{3}=0$

One may check that $P_{1}$ corresponds to the limit point and thus, the computation of the multiplicity only can be obtained from Theorem 2 or equivalently from Theorem 9 (see statements 2 and 3 of Remark 5) 
Since the point obtained in step 4 of the algorithm is $P_{1}$, we go to step 5 of the algorithm. In this step, we check whether

$$
P_{2}=\mathcal{P}(t, 8)=\left(3\left(6 t^{3}-2 t^{2}-2 t+1\right)(t+1) / t^{4},\left(t^{4}+20 t^{3}-20 t^{2}-5 t+5\right) / t^{4}, 8\right)
$$

is a singularity (see statement 4 in Remark 5). Note that $P_{2}$ parametrizes the line $-87+5 x_{1}-3 x_{2}=x_{3}-8=0$ (see Remark 6). For this purpose, we apply Lemma 1, and we get that $\operatorname{mult}\left(P_{2}, \mathcal{V}\right)=4$. Then, $\mathcal{S}=[\mathcal{G}, 4] \cup\left[P_{1}, 3\right] \cup\left[P_{2}, 4\right]$.

Now, we apply step 6 of the algorithm to check whether $Q_{1}=\mathcal{P}(0, t)=(0:-3$ : $-5: 0)$ is a singularity (see statement 5 in Remark 5). For this purpose, we apply Theorem 2 and Algorithm 2, and we get that $\operatorname{mult}\left(Q_{1}, \mathcal{V}\right)=4$. Hence,

$$
\mathcal{S}=[\mathcal{G}, 4] \cup\left[P_{1}, 3\right] \cup\left[P_{2}, 4\right] \cup\left[Q_{1}, 4\right] .
$$

For the other factor of the denominator of the parametrization, $m\left(t_{1}\right)=t_{1}^{3}-2 t_{1}^{2}+3 t_{1}-1$, we get that $Q_{2}:=\mathcal{G}=\{\overline{\mathcal{P}}(\bar{t})\}_{m\left(t_{1}\right)}=(0: 1: 0: 0)$ is a singularity of multiplicity 4 (see Remark 3 and statement 6 in Remark 5). Then,

$$
\mathcal{S}=[\mathcal{G}, 4] \cup\left[P_{1}, 3\right] \cup\left[P_{2}, 4\right] \cup\left[Q_{1}, 4\right] \cup\left[Q_{2}, 4\right] .
$$

Finally, we compute the missed line, L, of the parametrization $\overline{\mathcal{P}}$ by applying Theorem 2.6 in [26], and we get that $L$ is not a singularity. Thus, we return

$$
\mathcal{S}=[\mathcal{G}, 4] \cup\left[P_{1}, 3\right] \cup\left[P_{2}, 4\right] \cup\left[Q_{1}, 4\right] \cup\left[Q_{2}, 4\right] .
$$

In Example 4.2 of [14], elimination techniques has to be applied. In addition, in [14] the singularities are not explicitly computed and not all the singularities are provided (compare with Example 4.2. in [14]). We recall that the method presented in this paper does not introduce algebraic numbers during the computations.

\section{Proofs of Section 3}

In this section, we present the proofs of the results obtained in Section 3. In particular, we prove Propositions 1 and 2, Lemmas 1 and 2, and Theorems 7 and 8 .

Proposition 1. If $\mathcal{P}(\bar{t})$ is proper, then $\mathcal{P}_{t_{2}}^{*}\left(t_{1}\right)$ is proper.

Proof. By applying the results in Subsection 2.2, one has that $\operatorname{deg}\left(\psi_{\mathcal{P}_{t_{2}}^{*}}\right)=\operatorname{deg}_{t_{1}}(G)$, where $G\left(t_{1}, s_{1}, t_{2}\right)=\operatorname{gcd}\left(G_{1}\left(t_{1}, s_{1}, t_{2}\right), G_{2}\left(t_{1}, s_{1}, t_{2}\right), G_{3}\left(t_{1}, s_{1}, t_{2}\right)\right)$ (see (3)). Let us assume that $\operatorname{deg}\left(\psi_{\mathcal{P}_{t_{2}}^{*}}\right) \geq 2$. Thus, there exists $k\left(s_{1}\right) \in \bar{K}\left(s_{1}\right)$ (additionally to $s_{1}$ ) such that $G\left(k\left(s_{1}\right), s_{1}, t_{2}\right)=0$. Then, $\mathcal{P}\left(k\left(s_{1}\right), t_{2}\right)=\mathcal{P}\left(s_{1}, t_{2}\right)$ which implies that $\operatorname{deg}\left(\psi_{\mathcal{P}}\right) \geq 2$. This is impossible since $\mathcal{P}(\bar{t})$ is proper. 
Proposition 2. Let $P=\overline{\mathcal{P}}_{t_{2}}^{*}(s) \in \mathbb{P}^{2}\left(\overline{\mathbb{K}\left(t_{2}\right)}\right)$. If $P$ is an non-ordinary singularity of $\overline{\mathcal{C}}_{t_{2}}^{*}$, then $s \in \mathbb{K}$. In this case, $P=\left(c: a_{1}+t_{2} b_{1}: a_{2}+t_{2} b_{2}\right) \in \mathbb{P}^{2}\left(\mathbb{K}\left(t_{2}\right)\right), a_{i}, b_{i}, c_{i} \in \mathbb{K}$.

Proof. For the sake of completeness, we assume that $P$ is an affine point and we consider the affine parametrization $\mathcal{P}_{t_{2}}^{*}\left(t_{1}\right)$. For the case of an infinity point with the $j$-component non zero, one reasons analogously by using the dehomogenization of $\overline{\mathcal{P}}_{t_{2}}^{*}\left(t_{1}\right)$ w.r.t. the $j$-component. Thus, since $P$ is a non-ordinary singularity, two different situations appear:

- Let us assume that $\frac{\partial \mathcal{P}_{t_{2}}^{*}}{\partial t_{1}}(s)=(0,0)$. Hence

$$
p_{i 1}^{\prime}(s) q(s)-p_{i 1}(s) q^{\prime}(s)+t_{2}\left(p_{i 2}^{\prime}(s) q(s)-p_{i 2}(s) q^{\prime}(s)\right)=0, i=1,2 .
$$

Observe that if $p_{i 2}^{\prime}(s) q(s)-p_{i 2}(s) q^{\prime}(s)=0$, for some $i=1,2$, then $s \in \mathbb{K}$ (note that $\left.p_{i 2}^{\prime}\left(t_{1}\right) q\left(t_{1}\right)-p_{i 2}\left(t_{1}\right) q^{\prime}\left(t_{1}\right) \in \mathbb{K}\left[t_{1}\right]\right)$. Thus, we assume that $p_{i 2}^{\prime}(s) q(s)-p_{i 2}(s) q^{\prime}(s) \neq$ 0 , for $i=1,2$. Hence, $h(s)=0$, where

$$
\begin{gathered}
h\left(t_{1}\right)=\left(p_{11}^{\prime}\left(t_{1}\right) q\left(t_{1}\right)-p_{11}\left(t_{1}\right) q^{\prime}\left(t_{1}\right)\right)\left(p_{22}^{\prime}\left(t_{1}\right) q\left(t_{1}\right)-p_{22}\left(t_{1}\right) q^{\prime}\left(t_{1}\right)\right)- \\
\left(p_{21}^{\prime}\left(t_{1}\right) q\left(t_{1}\right)-p_{21}\left(t_{1}\right) q^{\prime}\left(t_{1}\right)\right)\left(p_{12}^{\prime}\left(t_{1}\right) q\left(t_{1}\right)-p_{12}\left(t_{1}\right) q^{\prime}\left(t_{1}\right)\right) .
\end{gathered}
$$

Since $h\left(t_{1}\right) \in \mathbb{K}\left[t_{1}\right]$, one concludes that $s \in \mathbb{K}$.

- Let us assume that there exist $s_{1}, s_{2} \in \overline{\mathbb{K}\left(t_{2}\right)}$, such that

$$
P=\mathcal{P}_{t_{2}}^{*}\left(s_{i}\right), i=1,2, \quad \frac{\partial \mathcal{P}_{t_{2}}^{*}}{\partial t_{1}}\left(s_{1}\right)=k\left(t_{2}\right) \frac{\partial \mathcal{P}_{t_{2}}^{*}}{\partial t_{1}}\left(s_{2}\right), k\left(t_{2}\right) \in \overline{\mathbb{K}\left(t_{2}\right)} .
$$

Then, the following equalities hold:

$$
\begin{aligned}
p_{11}\left(s_{1}\right)+t_{2} p_{12}\left(s_{1}\right) & =p_{11}\left(s_{2}\right)+t_{2} p_{12}\left(s_{2}\right), \\
p_{21}\left(s_{1}\right)+t_{2} p_{22}\left(s_{1}\right) & =p_{21}\left(s_{2}\right)+t_{2} p_{22}\left(s_{2}\right), \\
\left(p_{11}^{\prime}\left(s_{1}\right)+t_{2} p_{12}^{\prime}\left(s_{1}\right)\right)\left(p_{21}^{\prime}\left(s_{2}\right)+t_{2} p_{22}^{\prime}\left(s_{2}\right)\right) & =\left(p_{21}^{\prime}\left(s_{1}\right)+t_{2} p_{22}^{\prime}\left(s_{1}\right)\right)\left(p_{11}^{\prime}\left(s_{2}\right)+t_{2} p_{12}^{\prime}\left(s_{2}\right)\right) .
\end{aligned}
$$

From (7) and (8), we get that

$$
h_{1}\left(s_{1}, s_{2}\right)=0
$$

where

$h_{1}\left(t_{1}, t_{2}\right)=\left(p_{11}\left(t_{1}\right)-p_{11}\left(t_{2}\right)\right)\left(p_{22}\left(t_{2}\right)-p_{22}\left(t_{1}\right)\right)-\left(p_{12}\left(t_{2}\right)-p_{12}\left(t_{1}\right)\right)\left(p_{21}\left(t_{1}\right)-p_{21}\left(t_{2}\right)\right)$.

Note that if $p_{i j}^{\prime}\left(s_{k}\right)=0$, for some $i, j \in\{1,2\}$ and $k=1,2$, we get that $s_{k} \in \mathbb{K}$ and thus, from (10), we conclude that $s_{l} \in \mathbb{K}$, for $l=1,2, l \neq k$. Hence, we assume that $p_{i j}^{\prime}\left(s_{k}\right) \neq 0$, for $i, j \in\{1,2\}$ and $k=1,2$. 
From (9), we have that if $p_{21}^{\prime}\left(s_{1}\right)+t_{2} p_{22}^{\prime}\left(s_{1}\right)=0$, then $p_{21}^{\prime}\left(s_{2}\right)+t_{2} p_{22}^{\prime}\left(s_{2}\right)=0$ or $p_{11}^{\prime}\left(s_{1}\right)+t_{2} p_{12}^{\prime}\left(s_{1}\right)=0$. Since $p_{i j}^{\prime}\left(s_{k}\right) \neq 0$, if $p_{21}^{\prime}\left(s_{2}\right)+t_{2} p_{22}^{\prime}\left(s_{2}\right)=0$, we get that $p_{21}^{\prime}\left(s_{1}\right) p_{22}^{\prime}\left(s_{2}\right)-p_{21}^{\prime}\left(s_{2}\right) p_{22}^{\prime}\left(s_{1}\right)=0$. Thus, $f\left(s_{1}, s_{2}\right)=0$ with

$$
f\left(t_{1}, t_{2}\right)=p_{21}^{\prime}\left(t_{1}\right) p_{22}^{\prime}\left(t_{2}\right)-p_{21}^{\prime}\left(t_{2}\right) p_{22}^{\prime}\left(t_{1}\right) \in \mathbb{K}[\bar{t}] .
$$

Hence, from (10), we conclude that $s_{1}, s_{2} \in \mathbb{K}$ (one reasons similarly if $\left.p_{11}^{\prime}\left(s_{1}\right)+t_{2} p_{12}^{\prime}\left(s_{1}\right)=0\right)$.

Then, we assume that $p_{21}^{\prime}\left(s_{1}\right)+t_{2} p_{22}^{\prime}\left(s_{1}\right) \neq 0$ (and similarly $p_{21}^{\prime}\left(s_{2}\right)+t_{2} p_{22}^{\prime}\left(s_{2}\right) \neq$ $0)$. Thus, we may write (9) as

$$
\frac{p_{11}^{\prime}\left(s_{1}\right)+t_{2} p_{12}^{\prime}\left(s_{1}\right)}{p_{21}^{\prime}\left(s_{1}\right)+t_{2} p_{22}^{\prime}\left(s_{1}\right)}=\frac{p_{11}^{\prime}\left(s_{2}\right)+t_{2} p_{12}^{\prime}\left(s_{2}\right)}{p_{21}^{\prime}\left(s_{2}\right)+t_{2} p_{22}^{\prime}\left(s_{2}\right)} .
$$

Now, we derive w.r.t $t_{2}$ equalities (7) and (8), and we get that

$$
\left(p_{i 1}^{\prime}\left(s_{1}\right)+t_{2} p_{i 2}^{\prime}\left(s_{1}\right)\right) s_{1}^{\prime}+p_{i 2}\left(s_{1}\right)=\left(p_{i 1}^{\prime}\left(s_{2}\right)+t_{2} p_{i 2}^{\prime}\left(s_{2}\right)\right) s_{2}^{\prime}+p_{i 2}\left(s_{2}\right), i=1,2 .
$$

Let us assume that $s_{1}^{\prime} \neq 0$ (if $s_{i}=0$ for $i=1,2$, we get that $s_{i} \in \mathbb{K}$ for $i=1,2$ ). From (11) and (12), and taking into account that $p_{21}^{\prime}\left(s_{2}\right)+t_{2} p_{22}^{\prime}\left(s_{2}\right) \neq 0$, we get that

$$
\frac{p_{11}^{\prime}\left(s_{2}\right)+t_{2} p_{12}^{\prime}\left(s_{2}\right)}{p_{21}^{\prime}\left(s_{2}\right)+t_{2} p_{22}^{\prime}\left(s_{2}\right)}=\frac{\left(p_{11}^{\prime}\left(s_{2}\right)+t_{2} p_{12}^{\prime}\left(s_{2}\right)\right) s_{2}^{\prime}+\left(p_{12}\left(s_{2}\right)-p_{12}\left(s_{1}\right)\right)}{\left(p_{21}^{\prime}\left(s_{2}\right)+t_{2} p_{22}^{\prime}\left(s_{2}\right)\right) s_{2}^{\prime}+\left(p_{22}\left(s_{2}\right)-p_{22}\left(s_{1}\right)\right)}
$$

which implies that

$$
\left(p_{11}^{\prime}\left(s_{2}\right)+t_{2} p_{12}^{\prime}\left(s_{2}\right)\right)\left(p_{22}\left(s_{2}\right)-p_{22}\left(s_{1}\right)\right)=\left(p_{12}\left(s_{2}\right)-p_{12}\left(s_{1}\right)\right)\left(p_{21}^{\prime}\left(s_{2}\right)+t_{2} p_{22}^{\prime}\left(s_{2}\right)\right) .
$$

Let us assume that $p_{12}\left(s_{1}\right) \neq 0$ (note that if $p_{12}\left(s_{1}\right)=0$, then $s_{1} \in \mathbb{K}$ and thus, from $(10)$, we conclude that $\left.s_{2} \in \mathbb{K}\right)$, and we consider $g\left(t_{2}\right)=-\frac{p_{11}\left(s_{1}\right)}{p_{12}\left(s_{1}\right)}$. Let $\bar{s}_{i}=s_{i}\left(g\left(t_{2}\right)\right), i=1,2$, and let us prove that $\bar{s}_{i} \in \mathbb{K}, i=1,2$. For this purpose, we first note that if $p_{12}\left(\bar{s}_{2}\right)=0$, then $p_{11}\left(\bar{s}_{2}\right)=0$ which implies that $\bar{s}_{2} \in \mathbb{K}$ and then $\bar{s}_{1} \in \mathbb{K}$ (we substitute $t_{2}$ by $g\left(t_{2}\right)$ in $(10)$, and we get that $h_{1}\left(\bar{s}_{1}, \bar{s}_{2}\right)=0$ ). Thus, let us assume that $p_{12}\left(\bar{s}_{2}\right) \neq 0$. Then, from $(7)$, we get that $g\left(t_{2}\right)=-\frac{p_{11}\left(\bar{s}_{2}\right)}{p_{12}\left(\bar{s}_{2}\right)}$ and in this case, we also reach that $\bar{s}_{i} \in \mathbb{K}, i=1,2$. Indeed: by substituting $t_{2}$ by $g\left(t_{2}\right)=-\frac{p_{11}\left(s_{1}\right)}{p_{12}\left(s_{1}\right)}$ in $(10)$, we get that $h_{1}\left(\bar{s}_{1}, \bar{s}_{2}\right)=0$. Furthermore, by substituting $t_{2}$ by $g\left(t_{2}\right)=-\frac{p_{11}\left(s_{1}\right)}{p_{12}\left(s_{1}\right)}$ in $(13)$, and using that $g\left(t_{2}\right)=-\frac{p_{11}\left(\bar{s}_{2}\right)}{p_{12}\left(\bar{s}_{2}\right)}$, we get that $h_{2}\left(\bar{s}_{1}, \bar{s}_{2}\right)=0$, where

$$
\begin{gathered}
h_{2}\left(t_{1}, t_{2}\right)=\left(p_{11}^{\prime}\left(t_{2}\right) p_{12}\left(t_{2}\right)-p_{11}\left(t_{2}\right) p_{12}^{\prime}\left(t_{2}\right)\right)\left(p_{22}\left(t_{2}\right)-p_{22}\left(t_{1}\right)\right)- \\
\left(p_{12}\left(t_{2}\right)-p_{12}\left(t_{1}\right)\right)\left(p_{21}^{\prime}\left(t_{2}\right) p_{12}\left(t_{2}\right)-p_{11}\left(t_{2}\right) p_{22}^{\prime}\left(t_{2}\right)\right) .
\end{gathered}
$$


Thus, since $h_{i}\left(\bar{s}_{1}, \bar{s}_{2}\right)=0$, and $h_{i}\left(t_{1}, t_{2}\right) \in \mathbb{K}\left[t_{1}, t_{2}\right], i=1,2$, we get that $\bar{s}_{i} \in$ $\mathbb{K}, i=1,2$. Under these conditions, we deduce that

$$
g\left(t_{2}\right)=\frac{p_{11}\left(s_{1}\right)}{p_{12}\left(s_{1}\right)}=\frac{p_{11}\left(\bar{s}_{2}\right)}{p_{12}\left(\bar{s}_{2}\right)} \in \mathbb{K}
$$

which implies that $s_{1} \in \mathbb{K}$ and from (10), we conclude that $s_{2} \in \mathbb{K}$.

Lemma 1. It holds that $H(\beta)=0, \beta \in \mathbb{K}$, if and only if one of the following statements hold:

1. $\mathcal{P}\left(t_{1}, \beta\right) \in \mathbb{K}^{3}$. In this case, $\operatorname{mult}(P, \mathcal{V})=\operatorname{deg}(\mathcal{V})$, where $P:=\mathcal{P}(t, \beta)$.

2. $\mathcal{P}\left(t_{1}, \beta\right)=\left(a_{1}, a_{2}, \beta\right) \in \mathbb{K}\left(t_{1}\right)^{3} \backslash \mathbb{K}^{3}, a_{i} \in \mathbb{K}$, for some $i=1,2$. In this case, $\operatorname{mult}(P, \mathcal{V})=\operatorname{deg}(\mathcal{V})-\operatorname{deg}\left(q / m_{i}\right)$, where $P:=\mathcal{P}(t, \beta)$ and $q_{i}(\bar{t})-a_{i} q\left(t_{1}\right)=$ $\left(t_{2}-\beta\right) m_{i}\left(t_{1}\right)$ for some $i=1,2$.

3. (a) There exists $\alpha \in \mathbb{K}$ such that $q_{i}(\alpha, \beta)=q(\beta)=0, i=1,2$ (i.e. $\alpha$ is a base point of $\left.\mathcal{P}\left(t_{1}, \beta\right)\right)$ or

(b) $\mathcal{P}\left(t_{1}, \beta\right)$ is not proper. In this case, $\operatorname{mult}(P, \mathcal{V})=\operatorname{deg}\left(\psi_{\mathcal{P}\left(t_{1}, \beta\right)}\right)$, where $P:=$ $\mathcal{P}(t, \beta)$.

Proof. Taking into account the properties of the resultants (see e.g [25]), one has that $H(\beta)=0$ if and only if one of the following statements hold:

1. $\operatorname{lc}\left(\bar{G}_{i}, s_{1}\right)\left(t_{1}, \beta\right)=0$ for $i=1,2$. Then, since $\operatorname{deg}_{t_{1}}\left(q_{i}\right)=\operatorname{deg}(q)=d, i=1,2$, we get that

$$
a_{d} q_{i}\left(t_{1}, \beta\right)-q\left(t_{1}\right)\left(b_{d, i}+\beta c_{d, i}\right)=0, i=1,2,
$$

and thus $p_{i}\left(t_{1}, \beta\right)=\left(b_{d, i}+\beta c_{d, i}\right) / a_{d} \in \mathbb{K}, i=1,2$, where $a_{d}:=\operatorname{lc}_{t_{1}}(q) \in \mathbb{K} \backslash$ $\{0\}, b_{d, i}:=\mathrm{lc}_{t_{1}}\left(p_{i 1}\right) \in \mathbb{K}, c_{d, i}:=\mathrm{lc}_{t_{1}}\left(p_{i 2}\right) \in \mathbb{K}$, for $i=1,2$. Therefore, $\mathcal{P}\left(t_{1}, \beta\right) \in$ $\mathbb{K}^{3}$.

In this case, we prove that $\operatorname{mult}(P, \mathcal{V})=\operatorname{deg}(\mathcal{V})$, where $P:=\mathcal{P}(t, \beta)=$ $\left(a_{1}, a_{2}, \beta\right) \in \mathbb{K}^{3}$. Indeed: first, we observe that using Theorem 2 and that $\operatorname{deg}\left(\psi_{\mathcal{P}}\right)=1$, we have that $\operatorname{mult}(P, \mathcal{V})=\operatorname{deg}(\mathcal{V})-\operatorname{deg}\left(\psi_{\mathcal{R}_{P}}\right)$, where

$$
\mathcal{R}_{P}(\bar{t})=\left(\frac{p_{2}(\bar{t})-a_{2}}{p_{1}(\bar{t})-a_{1}}, \frac{t_{2}-\beta}{p_{1}(\bar{t})-a_{1}}\right)=\left(\frac{q_{2}(\bar{t})-a_{2} q\left(t_{1}\right)}{q_{1}(\bar{t})-a_{1} q\left(t_{1}\right)}, \frac{q\left(t_{1}\right)\left(t_{2}-\beta\right)}{q_{1}(\bar{t})-a_{1} q\left(t_{1}\right)}\right) .
$$

Note that $q_{i}(\bar{t})-a_{i} q\left(t_{1}\right)=\left(t_{2}-\beta\right) m_{i}\left(t_{1}\right), i=1,2$, and thus

$$
\mathcal{R}_{P}(\bar{t})=\left(\frac{m_{2}\left(t_{1}\right)}{m_{1}\left(t_{1}\right)}, \frac{q\left(t_{1}\right)}{m_{1}\left(t_{1}\right)}\right)
$$

which implies that $\operatorname{deg}\left(\psi_{\mathcal{R}_{P}}\right)=0$ (see Algorithm 2). Then, $\operatorname{mult}(P, \mathcal{V})=\operatorname{deg}(\mathcal{V})$. 
2. $\bar{G}_{i}\left(t_{1}, s_{1}, \beta\right)=0$ for some $i=1,2$. Let us assume that $\bar{G}_{1}\left(t_{1}, s_{1}, \beta\right)=0$ and $\bar{G}_{2}\left(t_{1}, s_{1}, \beta\right) \neq 0$. Thus $q_{1}\left(t_{1}, \beta\right) q\left(s_{1}\right)-q_{1}\left(s_{1}, \beta\right) q\left(t_{1}\right)=0$. Since $q\left(s_{1}\right) q\left(t_{1}\right) \neq$ 0 , we get that $p_{1}\left(t_{1}, \beta\right)=p_{1}\left(s_{1}, \beta\right)$ which implies that $p_{1}\left(t_{1}, \beta\right) \in \mathbb{K}$. Hence, $\mathcal{P}\left(t_{1}, \beta\right)=\left(a_{1}, a_{2}, \beta\right) \in \mathbb{K}\left(t_{1}\right)^{3}, a_{1} \in \mathbb{K}$.

In this case, we prove that $\operatorname{mult}(P, \mathcal{V})=\operatorname{deg}(\mathcal{V})-\operatorname{deg}\left(q / m_{1}\right)$, where $P:=$ $\mathcal{P}(t, \beta)=\left(a_{1}, a_{2}, \beta\right) \in \mathbb{K}(t)^{3}, a_{1} \in \mathbb{K}$ and $q_{1}(\bar{t})-a_{1} q\left(t_{1}\right)=\left(t_{2}-\beta\right) m_{1}\left(t_{1}\right)$. Indeed: we reason as in statement 1 and we get that $\operatorname{mult}(P, \mathcal{V})=\operatorname{deg}(\mathcal{V})-\operatorname{deg}\left(\psi_{\mathcal{R}_{P}}\right)$, where

$$
\mathcal{R}_{P}(\bar{t})=\left(\frac{p_{2}(\bar{t})-a_{2}}{p_{1}(\bar{t})-a_{1}}, \frac{t_{2}-\beta}{p_{1}(\bar{t})-a_{1}}\right)=\left(\frac{q_{2}(\bar{t})-a_{2} q\left(t_{1}\right)}{q_{1}(\bar{t})-a_{1} q\left(t_{1}\right)}, \frac{q\left(t_{1}\right)\left(t_{2}-\beta\right)}{q_{1}(\bar{t})-a_{1} q\left(t_{1}\right)}\right) .
$$

Note that $q_{1}(\bar{t})-a_{1} q\left(t_{1}\right)=\left(t_{2}-\beta\right) m_{1}\left(t_{1}\right)$, and thus

$$
\mathcal{R}_{P}(\bar{t})=\left(\frac{q_{2}(\bar{t})-a_{2} q\left(t_{1}\right)}{\left(t_{2}-\beta\right) m_{1}\left(t_{1}\right)}, \frac{q\left(t_{1}\right)}{m_{1}\left(t_{1}\right)}\right)
$$

which implies that $\operatorname{deg}\left(\psi_{\mathcal{R}_{P}}\right)=\operatorname{deg}\left(q / m_{1}\right)$ (note that since $\bar{G}_{2}\left(t_{1}, s_{1}, \beta\right) \neq 0$, then $p_{2}\left(t_{1}, \beta\right) \notin \mathbb{K}$ and thus $q_{2}\left(t_{1}, \beta\right)-a_{2}(\beta) q\left(t_{1}\right) \neq 0$; hence $\left(q_{2}(\bar{t})-a_{2} q\left(t_{1}\right)\right) /\left(\left(t_{2}-\right.\right.$ $\left.\left.\beta) m_{1}\left(t_{1}\right)\right) \in \mathbb{K}(\bar{t}) \backslash \mathbb{K}\left(t_{1}\right)\right)$. Therefore, $\operatorname{mult}(P, \mathcal{V})=\operatorname{deg}(\mathcal{V})-\operatorname{deg}\left(q / m_{1}\right)$.

For the case $\bar{G}_{2}\left(t_{1}, s_{1}, \beta\right)=0$ (and $\bar{G}_{1}\left(t_{1}, s_{1}, \beta\right) \neq 0$ ), we reason similarly. One only has to take into account that for this case, $q_{2}(\bar{t})-a_{2} q\left(t_{1}\right)=\left(t_{2}-\beta\right) m_{2}\left(t_{1}\right)$, and

$$
\mathcal{R}_{P}(\bar{t})=\left(\frac{\left(t_{2}-\beta\right) m_{2}\left(t_{1}\right)}{q_{1}(\bar{t})-a_{1} q\left(t_{1}\right)}, \frac{q\left(t_{1}\right)\left(t_{2}-\beta\right)}{q_{1}(\bar{t})-a_{1} q\left(t_{1}\right)}\right) .
$$

Observe that $\operatorname{deg}\left(\psi_{\mathcal{R}_{P}}\right)=\operatorname{deg}\left(\psi_{\mathcal{T}}\right)$, where

$$
\mathcal{T}(\bar{t})=\left(\frac{q_{1}(\bar{t})-a_{1} q\left(t_{1}\right)}{\left(t_{2}-\beta\right) m_{2}\left(t_{1}\right)}, \frac{q\left(t_{1}\right)}{m_{2}\left(t_{1}\right)}\right)
$$

$\left(\mathcal{R}_{P}=L(\mathcal{T})\right.$, where $L=(1 / x, y / x)$ is birational $)$. Thus, $\operatorname{deg}\left(\psi_{\mathcal{R}_{P}}\right)=\operatorname{deg}\left(q / m_{2}\right)$.

Finally, we note that $\bar{G}_{i}\left(t_{1}, s_{1}, \beta\right)=0$ for $i=1,2$ implies that $\mathcal{P}\left(t_{1}, \beta\right) \in \mathbb{K}^{3}$ and then, we are in statement 1 of the lemma.

3. There exists $\alpha \in \overline{\mathbb{K}\left(t_{1}\right)}$ such that $\bar{G}_{i}\left(t_{1}, \alpha, \beta\right)=0, i=1$, 2 , which implies that

$$
q_{i}\left(t_{1}, \beta\right) q(\alpha)-q_{i}(\alpha, \beta) q\left(t_{1}\right)=0, i=1,2 .
$$

We distinguish two different cases:

(a) Since $q\left(t_{1}\right) \neq 0$, if $q(\alpha)=0$ then $q_{i}(\alpha, \beta)=0, i=1,2$. This implies that $q_{i}(\alpha, \beta)=q(\beta)=0, i=1,2$. Then, $\alpha \in \mathbb{K}$ is a base point of $\mathcal{P}\left(t_{1}, \beta\right)$.

(b) If $q(\alpha) \neq 0$, then $\mathcal{P}(\alpha, \beta)=\mathcal{P}\left(t_{1}, \beta\right)$ which implies that $\operatorname{deg}\left(\psi_{\mathcal{P}\left(t_{1}, \beta\right)}\right) \geq 2$ (we have that $\alpha$ is a point of the fibre additionally to $t_{1}$; note that $\bar{G}_{i}\left(t_{1}, \alpha, \beta\right)=$ $0, i=1,2)$. Hence, $\mathcal{P}\left(t_{1}, \beta\right)$ is not proper. In this case, using statement 4 of Theorem 1, we get that $\operatorname{mult}(P, \mathcal{V})=\operatorname{deg}\left(\psi_{\mathcal{P}\left(t_{1}, \beta\right)}\right)$, where $P:=\mathcal{P}(t, \beta)$. 
Lemma 2. Let $\beta \in \mathbb{K}$ be such that $H(\beta) \neq 0$. It holds that, up to constants in $\mathbb{K} \backslash\{0\}$, $T\left(t_{1}, \beta\right)=T_{\beta}\left(t_{1}\right)$. In addition, $Q \in \mathbb{P}^{2}(\mathbb{K})$ is a singularity of $\overline{\mathcal{C}}_{\beta}^{*}$ of multiplicity $m$ if and only if $Q=P(\beta)$, where $P \in \mathbb{P}^{2}\left(\overline{\mathbb{K}\left(t_{2}\right)}\right)$ is a singularity of $\overline{\mathcal{C}}_{t_{2}}^{*}$ of multiplicity $m$.

Proof. First, we observe that $\operatorname{lc}\left(\bar{G}_{i}, s_{1}\right)\left(t_{1}, \beta\right) \neq 0$ for $i=1,2$. Otherwise, by applying Lemma 1, we get that $H(\beta)=0$ which is impossible.

Thus, we apply Lemma 4.3.1, p. 96 in [31], and we have that, up to constants in $\mathbb{K} \backslash\{0\}$,

$$
T\left(t_{1}, \beta\right)=T_{\beta}\left(t_{1}\right) .
$$

Then, from (5), (6) and (14), we deduce that,

$$
\begin{gathered}
\underbrace{\prod_{i=1}^{\ell} H_{P_{i}}\left(t_{1}, \beta\right)^{m_{i}-1} \prod_{i=\ell+1}^{k} h_{i 1}\left(t_{1}\right)^{r_{i 1}} \cdots h_{i m_{i}}\left(t_{1}\right)^{r_{i m_{i}}}}_{T\left(t_{1}, \beta\right)}= \\
\underbrace{\prod_{i=1}^{u} H_{Q_{i}}\left(t_{1}\right)^{n_{i}-1} \prod_{i=u+1}^{v} g_{i 1}\left(t_{1}\right)^{s_{i 1}} \cdots g_{i n_{i}}\left(t_{1}\right)^{s_{i n_{i}}}}_{T_{\beta}\left(t_{1}\right)}
\end{gathered}
$$

$\left(\right.$ recall that $\operatorname{gcd}\left(H_{P_{i}}, H_{P_{j}}\right)=\operatorname{gcd}\left(H_{Q_{i}}, H_{Q_{j}}\right)=1$, and $\left.\operatorname{gcd}\left(g_{i j}, g_{i k}\right)=\operatorname{gcd}\left(h_{i j}, h_{i k}\right)=1\right)$. Now, we observe that from Lemma 3 in [24], we have that for each $i=1, \ldots, k$, the polynomial $H_{P_{i}}\left(t_{1}, \beta\right)$ divides $H_{Q_{i}}\left(t_{1}\right)$ and $m_{i} \leq n_{i}, r_{i j} \leq s_{i j}$. Thus, up to constants in $\mathbb{K} \backslash\{0\}$,

$$
T_{\beta}\left(t_{1}\right)=\prod_{i=1}^{\ell}\left(H_{P_{i}}\left(t_{1}\right) M_{i}\left(t_{1}\right)\right)^{n_{i}-1} \prod_{i=\ell+1}^{k}\left(h_{i 1}\left(t_{1}\right) L_{i 1}\left(t_{1}\right)\right)^{s_{i 1}} \cdots\left(h_{i n_{i}}\left(t_{1}\right) L_{i n_{i}}\left(t_{1}\right)\right)^{s_{i n_{i}}} .
$$

Hence, equality (15) implies that $H_{P_{i}}\left(t_{1}, \beta\right)=H_{Q_{i}}\left(t_{1}\right), g_{i j}=h_{i j}, m_{i}=n_{i}$ and $r_{i j}=s_{i j}$. Therefore, if $Q \in \mathbb{P}^{2}(\mathbb{K})$ is a singularity of $\overline{\mathcal{C}}_{\beta}^{*}$ of multiplicity $m$ if and only if $Q=P(\beta)$, where $P \in \mathbb{P}^{2}\left(\overline{\mathbb{K}\left(t_{2}\right)}\right)$ is a singularity of $\overline{\mathcal{C}}_{t_{2}}^{*}$ of multiplicity $m$.

Theorem 7. The following statements hold:

1. Let $\left(a_{1}, a_{2}\right) \in{\overline{\mathbb{K}\left(t_{2}\right)}}^{2}$ be a singularity of $\mathcal{C}_{t_{2}}^{*}$ of multiplicity $m$. Then, $\left(a_{1}, a_{2}, t_{2}\right) \in$ ${\overline{\mathbb{K}}\left(t_{2}\right)}^{3}$ a singularity of $\mathcal{V}$ of multiplicity $m$.

2. Let $\left(\alpha_{1}, \alpha_{2}, \alpha_{3}\right) \in \mathbb{K}^{3}$ be a singularity of $\mathcal{V}$ of multiplicity $m$ such that $H\left(\alpha_{3}\right) \neq$ 0. Then, $\alpha_{i}=a_{i}\left(\alpha_{3}\right), i=1,2$, where $\left(a_{1}, a_{2}\right) \in{\overline{\mathbb{K}}\left(t_{2}\right)}^{2}$ a singularity of $\mathcal{C}_{t_{2}}^{*}$ of multiplicity $m$ 
Proof. 1. Let us denote by $f_{t_{2}}\left(x_{1}, x_{2}\right) \in\left(\mathbb{K}\left[t_{2}\right]\right)\left[x_{1}, x_{2}\right]$ the implicit polynomial defining the curve $\mathcal{C}_{t_{2}}^{*}$. In addition, let $g\left(x_{1}, x_{2}, x_{3}\right) \in \mathbb{K}\left[x_{1}, x_{2}, x_{3}\right]$ be the implicit polynomial defining the surface $\mathcal{V}$. Note that $f_{t_{2}}\left(x_{1}, x_{2}\right)=g\left(x_{1}, x_{2}, t_{2}\right)$, and

$$
\frac{\partial f_{t_{2}}}{\partial x_{1}^{d_{1}} \partial x_{2}^{d_{1}}}=\frac{\partial g}{\partial x_{1}^{d_{1}} \partial x_{2}^{d_{1}}}, d_{1}, d_{2} \in \mathbb{N}
$$

Furthermore, from $g\left(a_{1}, a_{2}, t_{2}\right)=0$, and taking into account that $\frac{\partial f_{t_{2}}\left(a_{1}, a_{2}\right)}{\partial x_{i}}=$ $0, i=1,2$, and (16), we get that

$$
\frac{\partial g\left(a_{1}, a_{2}, t_{2}\right)}{\partial x_{1}} a_{1}^{\prime}+\frac{\partial g\left(a_{1}, a_{2}, t_{2}\right)}{\partial x_{2}} a_{2}^{\prime}+\frac{\partial g\left(a_{1}, a_{2}, t_{2}\right)}{\partial x_{3}}=\frac{\partial g\left(a_{1}, a_{2}, t_{2}\right)}{\partial x_{3}}=0 .
$$

For the remaining partial derivatives, we reason similarly as above and using (16), we get statement 1.

2. In order to prove the second statement, we prove that $\left(\alpha_{1}, \alpha_{2}\right) \in \mathbb{K}^{2}$ a singularity of $\mathcal{C}_{\alpha_{3}}^{*}$ of multiplicity $m$. Thus, from Lemma 2, we obtain the second statement of the theorem. For this purpose, we first note that from (16), we get that $\left(\alpha_{1}, \alpha_{2}\right) \in \mathbb{K}^{2}$ a singularity of $\mathcal{C}_{\alpha_{3}}^{*}$ and $\operatorname{mult}\left(\left(\alpha_{1}, \alpha_{2}\right), \mathcal{C}_{\alpha_{3}}^{*}\right) \geq m$. Let us prove that the mult $\left(\left(\alpha_{1}, \alpha_{2}\right), \mathcal{C}_{\alpha_{3}}^{*}\right)=m$. Indeed: from Theorem 2, we have that $m=$ $\operatorname{deg}(\mathcal{V})-\operatorname{deg}\left(\psi_{\mathcal{R}_{P}}\right)$, where

$$
\mathcal{R}_{P}(\bar{t})=\left(\frac{p_{2}(\bar{t})-\alpha_{2}}{p_{1}(\bar{t})-\alpha_{1}}, \frac{t_{2}-\alpha_{3}}{p_{1}(\bar{t})-\alpha_{1}}\right) .
$$

In addition, using Remark 1 and that $\mathcal{P}_{\alpha_{3}}^{*}\left(t_{1}\right)$ is a proper parametrization (see Lemma 1), we have that

$$
\operatorname{mult}\left(\left(\alpha_{1}, \alpha_{2}\right), \mathcal{C}_{\alpha_{3}}^{*}\right)=\operatorname{deg}\left(\mathcal{C}_{\alpha_{3}}^{*}\right)-\operatorname{deg}\left(\frac{p_{2}\left(t_{1}, \alpha_{3}\right)-\alpha_{2}}{p_{1}\left(t_{1}, \alpha_{3}\right)-\alpha_{1}}\right) .
$$

Observe that

$$
\operatorname{deg}(\mathcal{V}) \geq \operatorname{deg}\left(\mathcal{C}_{t_{2}}^{*}\right) \geq \operatorname{deg}\left(\mathcal{C}_{\alpha_{3}}^{*}\right), \text { and } \operatorname{deg}\left(\psi_{\mathcal{R}_{P}}\right) \geq \operatorname{deg}\left(\frac{p_{2}\left(t_{1}, \alpha_{3}\right)-\alpha_{2}}{p_{1}\left(t_{1}, \alpha_{3}\right)-\alpha_{1}}\right) .
$$

Therefore, $\operatorname{mult}\left(\left(\alpha_{1}, \alpha_{2}\right), \mathcal{C}_{\alpha_{3}}^{*}\right) \leq m$. Since we had that $\operatorname{mult}\left(\left(\alpha_{1}, \alpha_{2}\right), \mathcal{C}_{\alpha_{3}}^{*}\right) \geq m$, we conclude that mult $\left(\left(\alpha_{1}, \alpha_{2}\right), \mathcal{C}_{\alpha_{3}}^{*}\right)=m$.

Theorem 8. Let $\left(0: a_{1}: a_{2}\right) \in \mathbb{P}^{2}\left(\overline{\mathbb{K}\left(t_{2}\right)}\right)$ be a singularity of $\overline{\mathcal{C}}_{t_{2}}^{*}$ of multiplicity $m$. Then, $P:=\left(0: a_{1}: a_{2}: 0\right) \in \mathbb{P}^{3}\left(\overline{\mathbb{K}\left(t_{2}\right)}\right)$ is a singularity of $\overline{\mathcal{V}}$ and

$$
\operatorname{mult}(P, \overline{\mathcal{V}})=\operatorname{deg}(\mathcal{V})-\operatorname{deg}_{t_{1}}\left(\frac{a_{2} q_{1}(\bar{t})-a_{1} q_{2}(\bar{t})}{q\left(t_{1}\right)}\right) \geq m
$$


Proof. First, let us assume that $a_{2} \neq 0$, and thus the given infinity point can be written as $(0: a: 1) \in \mathbb{P}^{2}\left(\overline{\mathbb{K}\left(t_{2}\right)}\right)$. Hence, we apply Remark 1 using the dehomogenization of $\overline{\mathcal{P}}_{t_{2}}^{*}\left(t_{1}\right)$ w.r.t. the 3 -component, and we get that,

$$
\operatorname{mult}\left((0: a: 1), \overline{\mathcal{C}}_{t_{2}}^{*}\right)=\operatorname{deg}\left(\overline{\mathcal{C}}_{t_{2}}^{*}\right)-\operatorname{deg}_{t_{1}}\left(\frac{q_{1}(\bar{t})-a q_{2}(\bar{t})}{q\left(t_{1}\right)}\right)
$$

(note that from Proposition $1, \operatorname{deg}\left(\psi_{\mathcal{P}_{t_{2}}^{*}}\right)=1$.) Now, taking into account that $\mathcal{P}$ is proper, and applying Theorem 2 (we use the dehomogenization of $\overline{\mathcal{P}}(\bar{t})$ w.r.t. the 3-component), we get that,

$$
\operatorname{mult}((0: a: 1: 0), \overline{\mathcal{V}})=\operatorname{deg}(\overline{\mathcal{V}})-\operatorname{deg}\left(\psi_{\mathcal{R}_{P}}\right), \text { where } \mathcal{R}_{P}(\bar{t})=\left(\frac{q_{1}(\bar{t})-a q_{2}(\bar{t})}{q\left(t_{1}\right)}, t_{2}\right) .
$$

Then $\operatorname{deg}\left(\psi_{\mathcal{R}_{P}}\right)=\operatorname{deg}_{t_{1}}\left(\left(q_{1}(\bar{t})-a q_{2}(\bar{t})\right) / q\left(t_{1}\right)\right)$, and since $\operatorname{deg}\left(\overline{\mathcal{C}}_{t_{2}}^{*}\right) \leq \operatorname{deg}(\overline{\mathcal{V}})$, we get

$$
\operatorname{mult}(P, \overline{\mathcal{V}})=\operatorname{deg}(\overline{\mathcal{V}})-\operatorname{deg}_{t_{1}}\left(\frac{q_{1}(\bar{t})-a q_{2}(\bar{t})}{q\left(t_{1}\right)}\right) \geq m
$$

If $a_{2}=0$ then, the given infinity point is $(0: 1: 0) \in \mathbb{P}^{2}(\mathbb{K})$. Hence, we apply Remark 1 using the dehomogenization of $\overline{\mathcal{P}}_{t_{2}}^{*}\left(t_{1}\right)$ w.r.t. the 2-component, and we get that, $\operatorname{mult}\left((0: 1: 0), \overline{\mathcal{C}}_{t_{2}}^{*}\right)=\operatorname{deg}\left(\overline{\mathcal{C}}_{t_{2}}^{*}\right)-\operatorname{deg}_{t_{1}}\left(q_{2}(\bar{t}) / q\left(t_{1}\right)\right)$. Now, taking into account that $\mathcal{P}$ is proper, and applying Theorem 2 (we use the dehomogenization of $\overline{\mathcal{P}}(\bar{t}$ ) w.r.t. the 2-component), we get that, $\operatorname{mult}((0: 1: 0: 0), \overline{\mathcal{V}})=\operatorname{deg}(\overline{\mathcal{V}})-\operatorname{deg}\left(\psi_{\mathcal{R}_{P}}\right)$, where $\mathcal{R}_{P}(\bar{t})=\left(q_{2}(\bar{t}) / q\left(t_{1}\right), t_{2}\right)$. Observe that $\operatorname{deg}\left(\psi_{\mathcal{R}_{P}}\right)=\operatorname{deg}_{t_{1}}\left(q_{2}(\bar{t}) / q\left(t_{1}\right)\right)$, and since $\operatorname{deg}\left(\overline{\mathcal{C}}_{t_{2}}^{*}\right) \leq \operatorname{deg}(\overline{\mathcal{V}})$, we get that

$$
\operatorname{mult}((0: 1: 0: 0), \overline{\mathcal{V}})=\operatorname{deg}(\overline{\mathcal{V}})-\operatorname{deg}_{t_{1}}\left(q_{2}(\bar{t}) / q\left(t_{1}\right)\right) \geq m .
$$

Thus, from both cases we deduce the theorem.

\section{Conclusions}

Given an algebraic variety implicitly defined, the singularities and their multiplicities can be directly computed by applying elimination theory techniques as for instance, Gröbner bases. When the variety is defined by means of a rational parametrization, besides the question of computing the singular locus and its multiplicity structure, one has the additional problem of determining the parameter values that generate the singular points with their corresponding multiplicities. This, for instance, can be useful when using a parametrization for plotting a curve or a surface or when utilizing a parametrization for analyzing the intersection variety of two varieties being one of them parametrically given. 
For the parametric case, one can always apply elimination techniques to first provide the defining implicit polynomials of the variety, second to determine the singularities from these polynomials, third to decompose the singular locus w.r.t. the multiplicities, and finally to compute the fibre (w.r.t. the parametrization) of the elements in the singular locus. Nevertheless, this is inefficient because of the computational complexity. So, for the parametric case, the challenge is to derive the singularities and their multiplicity directly from a parametric representation avoiding the computation of the ideal of the variety.

The case of rational curves has been efficiently addressed by several authors (see e.g. [2], [7], [11], [19]). For the case of surfaces, in [23], authors focus on the problem of computing the singularities, as well as their multiplicities, of rational surfaces given parametrically. In [14], a particular algorithm is presented to compute the selfintersection curves of a rational ruled surface based on the theory of $\mu$-bases. However, in these two papers, elimination techniques have to be finally applied and algebraic numbers have to be introduced during the computations. In addition, in [14] the singularities are not explicitly computed and not all the singularities are provided (see Example 4 to compare these two methods).

The approach presented in this paper improves the previous algorithms for detecting singularities (for the special case of parametric ruled surfaces). This approach is based on the computation of the singularities (and their multiplicities) of an auxiliary parametric curve directly defined from the given rational parametrization of the surface. We prove that the singularities of this auxiliary curve provide the singularities of the input ruled surface and only a finite set of points have to be additionally analyzed. In this way, the problem of the computation of singularities for a given parametric ruled surface is greatly simplified. The algorithm presented is very efficient since it is based on the computation of a unique univariate resultant. The approach developed in this paper follows from the results obtained in [2], [7], [3], [4], [19], where a complete classification of the singularities (and their multiplicities) of a given curve, via the factorization of a univariate resultant, is obtained.

As a future work, it would be interesting to use this method to compute the singular locus of a more general rational surfaces. For instance, one could analyze whether the method presented in Section 3 can be generalized for rational surfaces over $\mathbb{K}$ that admit a rational parametrization $\mathcal{P}(\bar{t})=\left(p_{1}(\bar{t}), p_{2}(\bar{t}), t_{2}\right)$, where $p_{i}(\bar{t}) \in \mathbb{K}(\bar{t}), i=1,2$. Currently there are no complete results for these general surfaces and further study is necessary.

\section{Acknowledgements}

This work has been partially supported by the Spanish Ministerio de Ciencia, Innovación y Universidades and by the European Regional Development Fund (ERDF), 
under the project MTM2017-88796-P. Author belongs to the Research Group ASYNACS (Ref. CCEE2011/R34).

\section{References}

[1] Andradas C., Recio R., Sendra J.R., Tabera L.F., Villarino C. (2011). Proper real reparametrization of rational ruled surfaces. Computer Aided Geometric Desig. Vol. 28, pp. 102-113.

[2] Blasco A., Pérez-Díaz S. (2017). Resultants and Singularities of Parametric Curves. See arXiv:1706.08430v3 [math.AG]. Submitted to Journal of Algebra and its Applications.

[3] Blasco A., Pérez-Díaz S. (2018). The Limit Point and the T-Function. Journal of Symbolic Computation. DOI: 10.1016/j.jsc.2018.06.009. To appear.

[4] Blasco A., Pérez-Díaz S. (2018). A Resultant Based Method for Studying the NonOrdinary Singularities of a Rational Curve. Computer Aided Geometric Design. To appear.

[5] Busé L., Elkadi M., Galligo A. (2009). A computational study of ruled surfaces. Journal of Symbolic Computation. Vol. 44, pp. 232-241.

[6] Busé, L., Thang, L.B. (2010) Matrix-based implicit representations of rational algebraic curves and applications. Computer Aided Geometric Design 27 vol. 9, 681-699.

[7] Busé, L., D'Andrea, C. (2012) Singular factors of rational plane curves. Journal of Algebra. Vol. 357 , pp. 322-346.

[8] Chen F. (2003). Reparametrization of a rational ruled surface using the $\mu$-Basis. Computer Aided Geometric Design. Vol. 20, pp. 11-17.

[9] Chen F., Zheng J., Sederberg T.W. (2001). The $\mu$-Basis of a rational ruled surface. Computer Aided Geometric Design. Vol. 18, pp. 61-72.

[10] Chen Y., Shen L.-Y., Yuan C. (2011). Collision and intersection detection of two ruled surfaces using bracket method. Computer Aided Geometry Design. Vol.28, pp. 114-126.

[11] Chen F., Wang W.P. Liu, Y. (2008). Computing singular points of plane rational curves. Journal of Symbolic Computation 43 (2), 92-117.

[12] Dohm M. (2009). Implicitization of rational ruled surfaces with $\mu$-Bases. Journal of Symbolic Computation. Vol. 44, pp. 479-489.

[13] Harris J. (1995). Algebraic Geometry. A first Course. Springer-Verlag.

[14] Jia X., Chen F. Deng J. (2009). Computing self-intersection curves of rational ruled surfaces. Computer Aided Geometric Design. Vol. 26. pp. 287-299

[15] Izumiya S., Takeuchi N. (2003). Special curves and ruled surfaces. Contributions to Algebra and Geometry. Vol. 44(1), pp. 203-212.

[16] Li J., Shen L.-Y., Gao X.-S. (2008). Proper reparametrization of ruled surface. Journal of Comput. Sci. and Tech. Vol. 5, pp. 290-297.

[17] Liu Y., Pottmann H., Wallner J., Yang Y., Wang W. (2006). Geometric modeling with conical meshes and developable surfaces. ACM Transactions on Graphics. Vol. 25(3), pp. 1-9.

[18] Pérez-Díaz S. (2006). On the Problem of Proper Reparametrization for Rational Curves and Surfaces. Computer Aided Geometric Design. Vol. 23/4, pp. 307-323.

[19] Pérez-Díaz, S. (2007). Computation of the Singularities of Parametric Plane Curves. Journal of Symbolic Computation. Vol. 42/8. pp. 835-857.

[20] Pérez-Díaz S., Sendra J.R. (2004). Computation of the degree of rational surface parametrizations. Journal of Pure and Applied Algebra. Vol. 193(1-3), pp. 99-121.

[21] Pérez-Díaz S., Sendra J.R. (2005). Partial Degree Formulae for Rational Algebraic Surfaces. Proc. ISSAC05 pp. 301-308. ACM Press.

[22] Pérez-Díaz S., Sendra J.R. (2008). A Univariate Resultant Based Implicitization Algorithm for Surfaces. Journal of Symbolic Computation. Vol. 43, pp. 118-139.

[23] Pérez-Díaz S., Sendra J.R., Villarino C. (2015). Computing the Singularities of Rational Surfaces. Mathematics of Computation. Volume 84, Number 294, pp. 1991-2021. 
[24] Sendra J.R., Winkler F. (2001). Tracing index of rational curve parametrizations. Comput. Aided Geom. Des. Vol. 18 (8). pp. 771-795.

[25] Sendra J.R., Winkler F., Pérez-Díaz S. (2007). Rational Algebraic Curves: A Computer Algebra Approach. Series: Algorithms and Computation in Mathematics. Vol. 22. Springer Verlag.

[26] Sendra J.R., Sevilla D., Villarino C. (2017). Covering rational ruled surfaces. Math. Comp. Vol. 86, pp. 2861-2875.

[27] Shafarevich I.R. (1994). Basic algebraic geometry Schemes; 1 Varieties in projective space. Berlin New York: Springer-Verlag.

[28] Shen L.-Y., Cheng J., Jia X.(2012). Homeomorphic approximation of the intersection curve of two rational surfaces. Computer Aided Goemetric Design, Vol 29(8), pp. 613-625.

[29] Shen L.-Y., Pérez-Díaz S. (2014). Characterization of Rational Ruled Surfaces. Journal of Symbolic Computation. Volume 63, pp. 21-45.

[30] Wang X., Goldman R. (2015). Quaternion rational surfaces: Rational surfaces generated from the quaternion product of two rational space curves. Graphical Models. Volume 81, pp. 18-32.

[31] Winkler F., (1996). Polynomial Algorithms in Computer Algebra. Springer-Verlag, Wien New York. 